\section{International Scientific Journal Theoretical \& Applied Science}

p-ISSN: 2308-4944 (print) e-ISSN: 2409-0085 (online)

Year: 2018 Issue: 09 Volume: 65

Published: $29.09 .2018 \quad$ http://T-Science.org

SECTION 2. Applied mathematics. Mathematical modeling.

\section{S.U. Zhanatauov}

candidate of physics and mathematical sciences,

Department «Information technologies and automation», Associate professor, Noncommercial joint-stock company "Kazakh national agrarian university"

Kazakhstan sapagtu@mail.ru

K.A. Akhmetov candidate of technical sciences, Department «Information technologies and automation», Professor, Noncommercial joint-stock company "Kazakh national agrarian university"

Kazakhstan

kahmetov@mail.ru

\title{
SIMULATION OF MULTIDIMENSIONAL SAMPLE WITH THE ASSIGNED VALUES OF THE COEFFICIENTS OF LINEAR REGRESSION
}

\begin{abstract}
The article describes the calculation steps for model values of the matrix elements of centered and normalized values of $z$-variables $\left.Z_{m n}=\left[Z_{1}\right\rfloor Z_{2}\right]$. The partition $\left[Z_{1} \mid Z_{2}\right]$ corresponds to a regression model of the form $z_{n}=\beta_{1} z_{1}+\beta_{2} z_{2}+\ldots+\beta_{n-} z_{n}=\beta_{1} z_{1}+\beta_{2 z_{2}}+\ldots+\beta_{n-1}$, where $z_{1}, z_{2}, \ldots, z_{n-1}$ is a set of explanatory (independent) variable ("regressors"), $z_{n}$ - response variable (dependent variable), $\beta_{1}, \ldots, \beta_{n-1}$-regression coefficients. There is an adequacy of the real multi-dimensional sample $X^{0}{ }_{m n}$ as a model, histogram, and the mean arithmetic mean $x^{m e}$, standard deviations $s_{j}=\operatorname{sqrt}\left(s^{2}\right), j=1, \ldots, n$. The tables, the graphs of the parameters, the variables of the IM MLRA are given for $m=20, n=3$ :

$Z^{\left(t, \ell,{ }_{m n}\right.}=\left[Z^{(t, \ell,)}{ }_{1} / Z^{(t, \ell)}{ }_{2}\right],(1 \mathrm{Vm}) Z^{(t) T}{ }_{1} Z^{(t)}{ }_{1}=R^{(\ell)}{ }_{11},(1 \mathrm{Vm}) Z^{(t) T}{ }_{1} Z^{(\ell, t)}{ }_{2}=R^{(\ell)}{ }_{12}, R^{(\ell)}{ }_{12}=R^{(\ell)}{ }_{11} \beta, \beta=\left(\beta_{1}, \beta_{2}\right)^{T}$.

Key words: inverse model, multidimensional, linear, regression analysis.

Language: Russian

Citation: Zhanatauov SU, Akhmetov KA (2018) SIMULATION OF MULTIDIMENSIONAL SAMPLE WITH THE ASSIGNED VALUES OF THE COEFFICIENTS OF LINEAR REGRESSION. ISJ Theoretical \& Applied Science, 09 (65): 301-314.

Soi: http://s-o-i.org/1.1/TAS-09-65-48 Doi: crossef https://dx.doi.org/10.15863/TAS.2018.09.65.48

\section{МОДЕЛИРОВАНИЕ МНОГОМЕРНОЙ ВЫБОРКИ С ЗАДАННЫМИ ЗНАЧЕНИЯМИ КОЭФФИЦИЕНТОВ ЛИНЕЙНОЙ РЕГРЕССИИ}

Аннотация: В статье дано описание этапов вычислений модельных значений элементов матриць центрированных и нормированных значений z-переменных $\left.Z_{m n}=\left[Z_{1}\right\rfloor Z_{2}\right]$. Матрица $Z_{m n}=\left[Z_{1} \mid Z_{2}\right]$. Разбиение $\left.\left[Z_{1}\right\rfloor Z_{2}\right]$ соответствует регрессионной модели вида $z_{n}=\beta_{1} z_{1}+\beta_{2} z_{2}+\ldots+\beta_{n-1} z_{n-1}, \quad$ где $z_{1}, z_{2}, \ldots, z_{n-1}-$ набор объясняющих (независимых) переменных («регрессоров»), $z_{n}$-переменная отклика (зависимая переменная), $\beta_{1}, \ldots, \beta_{n-1^{-}}$регрессионные коэффициенты. Имеется адекватность реальной многомерной выгорке $X^{0}{ }_{\text {mn }}$ как модельная, гистограммная, так и по значениям средних арифметических $x^{c p}{ }_{j}=x^{c p}$, стандартных отклонений $s_{j}=\operatorname{sqrt}\left(s_{j}^{2}\right), j=1, \ldots, n$. Приведень таблищь, графики параметров, переменньх ОМ МЛРА при $m=20, n=3: Z^{(t, \ell,)}{ }_{m n}=\left[Z^{(t, \ell,)}{ }_{1} \mid Z^{(t, \ell)}{ }_{2}\right],(I \mathrm{Vm}) Z^{(t) T}{ }_{1} Z^{(t)}{ }_{1}=R^{(\ell)}{ }_{11},(I \mathrm{Vm}) Z^{(t) T}{ }_{1} Z^{(\ell, t)}{ }_{2}=R^{(\ell)}{ }_{12}, R^{(\ell)}{ }_{12}=R^{(\ell)}{ }_{11} \beta, \beta=\left(\beta_{1}, \beta_{2}\right)^{T}$.

Ключевые слова: обратная модель, множественный, линейный, регрессионный анализ.
\end{abstract}

\section{Введение}

Регрессионные модели разработаны в многочисленных вариантах, применялись во многих предметных областях. Многочисленные статьи, монографии на разных языках, 
программы для ЭВМ общеизвестны, стали повседневным инструментарием.

Представляет интерес последовательность значений компонент вектора значений коэффициентов регрессии, начиная с «нехороших» кончая желаемыми. Значения компонент вектора значений коэффициентов регрессии $\beta_{1}, \beta_{2}, \ldots, \beta_{\mathrm{n}-1}$ будут вычислены, если имеются числовые данные. Мы рассматриваем в качестве данных матрицу центрированных и нормированных значений $\mathrm{Z}_{\mathrm{mn}}=\left[\mathrm{Z}_{1} \mid \mathrm{Z}_{2}\right]$. Рассмотрим регрессионную модель вида $\mathrm{z}_{\mathrm{n}}=\beta_{1} \mathrm{z}_{1}+\beta_{2} \mathrm{z}_{2}+\ldots+\beta_{\mathrm{n}-1} \mathrm{z}_{\mathrm{n}-1}+\alpha$, где $\mathrm{z}_{1}, \mathrm{z}_{2}, \ldots, \mathrm{z}_{\mathrm{n}-1}$-набор объясняющих (независимых) переменных («регресссоров»), $\quad \mathrm{Z}_{\mathrm{n}}$-переменная отклика (зависимая переменная), $\beta_{1}, \ldots, \beta_{\mathrm{n}-1-}$ регресссионные коэффициенты, $\alpha$-свободный член. Эта модель моделирует взаимосвязь между двумя или более объясняющими переменными и одной переменной отклика путем подгонки вышеприведенного линейного уравнения к стандартизованным значениям z-переменных $\mathrm{z}_{\mathrm{ij}}=\left(\mathrm{x}_{\mathrm{ij}}^{0}-\mathrm{X}^{\mathrm{cp}} \mathrm{j}_{\mathrm{j}}\right) / \mathrm{s}_{\mathrm{j}}$. Здесь $\mathrm{x}_{\mathrm{ij}}^{0}$ - i-ое значение $\mathrm{j}$-го признака реального объекта, $\mathrm{x}^{\mathrm{cp}}=\left(\mathrm{x}^{0}{ }_{1 \mathrm{j}}+\ldots+\mathrm{x}^{0}{ }_{\mathrm{mj}}\right) / \mathrm{m}$ среднее арифметическое, $\mathrm{s}_{\mathrm{j}} \mathrm{j}=\left(\mathrm{x}^{2}{ }_{1 \mathrm{j}}+\ldots+\mathrm{x}_{\mathrm{mj}}{ }^{2}\right) / \mathrm{m}$ стандартное отклонение, $\mathrm{x}_{\mathrm{ij}}=\mathrm{x}_{\mathrm{ij}}^{0} \mathrm{X}^{\mathrm{cp}}{ }_{\mathrm{j}}$ - отклонение от среднего значения $\mathrm{x}^{\mathrm{cp}}$. Стандартизованные значения $Z_{n}$ изменяются относительно значений $\mathrm{Z}_{1}, \mathrm{Z}_{2}, \ldots, \mathrm{Z}_{\mathrm{n}-1}$ с одинаковыми стандартными отклонениями, равными 1 . В соответствии с этим разбиением z-переменных $\mathrm{m}$ значений всех n zпеременных образует 2 подматрицы $Z_{1}, Z_{2}$ матрицы $Z_{m n}=\left[Z_{1} \mid Z_{2}\right]$ для m-на-n матрицы $Z_{m n}$. Элементы столбцов (с номерами $\mathrm{j}=1, \ldots, \mathrm{n}$ ) матрицы $Z_{\mathrm{mn}}$ центрированы выборочными средними и нормированы стандартными отклонениями: $\mathrm{z}_{\mathrm{ij}}=\left(\mathrm{x}_{\mathrm{ij}}^{0} \mathrm{X}^{\mathrm{cp}} \mathrm{j}\right) / \mathrm{s}_{\mathrm{j}}$. Элементы $\mathrm{z}_{\mathrm{ij}}=\left(\mathrm{x}_{\mathrm{ij}}{ }^{-}\right.$ $\left.\mathrm{x}^{\mathrm{cp}}{ }_{\mathrm{j}}\right) / \mathrm{s}_{\mathrm{j}}$ матрицы стандартизованных отклонений не имеют размерности, и все ее столбцы имеют одинаковые дисперсии, равные единице. Это одно из удобств для наших задач.

Однако, в связи с кризисными событиями актуальны задачи управления вычисленными значениями коэффициентов регрессии $\beta_{1}, \ldots, \beta_{\mathrm{n}-1}$, изменяя их значения $\beta^{(t)} 1, \ldots, \beta^{(t)}{ }_{n-1}$ в момент времени $\mathrm{t}$ на заданные экспертом значения. Изменения проводятся от отрицательных значений (от убыточных приращений в уравнении регрессии) к положительным значениям (к прибыли). Величина приращения к ј-ому значению $\beta_{\mathrm{j}}$ назначается экспертом отдельно и требует соответствующей работы. В примере, описываемом ниже, приведены результаты только начальных значений и конечных значений коэффициентов регрессии $\beta_{1}, \ldots, \beta_{\mathrm{n}-1}$.

Мы рассматриваем в качестве исходных значения $\beta_{1}, \ldots, \beta_{\mathrm{n}-1}$ и моделируем для них матрицу $\mathrm{Z}_{\mathrm{mn}}=\left[\mathrm{Z}_{1} \mid \mathrm{Z}_{2}\right]$ значений $\mathrm{n}$ Z-переменных, такую, что она имеет значения $\beta_{1}, \ldots, \beta_{\mathrm{n}-1}$. Таких матриц моделируются бесконечно много, каждая преобразуется в псевдореальную выборку данных с применением своих средних и стандартных отклонений (Рисунок 7).

Например, прибыль (зависимая переменная или отклик) в зависимости от прироста ресурсов, вложений (независимых переменных) в прибыльные активные операции. Предварительная фиксация значений коэффициентов регрессии $\beta_{1}, \ldots, \beta_{\mathrm{n}-1}$ и моделирование значений независимых и зависимой переменных позволит иметь ряды матриц $Z_{\mathrm{mn}}=\left[\mathrm{Z}_{1} \mid \mathrm{Z}_{2}\right]$, достигающих по построению такой матрицы $Z_{\mathrm{mn}}=\left[Z_{1} \mid Z_{2}\right]$, которая будет иметь заданные целевые значения коэффициентов регрессии $\quad \beta_{1}, \ldots, \beta_{\mathrm{n}-1}$. Тогда возможно проектирование рядов векторов значений коэффициентов регрессии $\boldsymbol{\beta}=\left(ß_{1}, \ldots, \beta_{\mathrm{n}-1}\right)^{\mathrm{T}}$ и соответствующих им рядов матриц $Z_{\mathrm{mn}}=\left[Z_{1} \mid Z_{2}\right]$ c заданными свойствами. Предыдущий и последующий члены ряда векторов значений коэффициентов регрессии нужно подбирать вручную и в соответствии с реальными производственными, маркетинговыми, административными мероприятия ми, обеспечивающих достижение планируемых финансовых приростов значений ресурсов, вложений. Если нет реального менеджмента по достижению планируемых значений $\beta_{1}, \ldots, \beta_{\mathrm{n}-1}$, то нет пользы от модельных значений элементов матрицы $Z_{m n}=\left[Z_{1} \mid Z_{2}\right]$. Матрица $Z_{m n}=\left[Z_{1} \mid Z_{2}\right]$ при известных значениях средних и стандартных отклонений преобразуются в «реальные» значения с единицами измерения показателей с номерами $\mathrm{j}=1, \ldots, n$. Каждая цифра в «реальной» матрице адекватна реальному значению, если значения n z-переменных удовлетворяют системе уравнений (*). При этом практические решения по принятию предыдущего значения и последующего значения должны быть подвергнуты всестороннему анализу.

Современные тенденции в теории и практике финансового анализа связаны с проблемой модификации системы финансовых коэффициентов, с приведением этой системы к форме, удобной для принятия адекватных управленческих решений в области финансового менеджмента. В этом направлении существует несколько подходов. Предпочтителен подход, когда выбирают из всех существующих финансовых показателей и коэффициентов незначительное количество тех, которые наиболее полно и всесторонне характеризуют финансовое состояние банка.

Здесь мы остановимся на статистическом подходе к коэффициентному методу финансового анализа. Суть нашего подхода может быть сведена к анализу выборочных коэффициентов корреляции и коэффициентов 
регрессии. Последние имеют практически важные смысл и интерпретацию: «если банк увеличит на 1 тысячу тенге свои кредитные вложения, то банк потерпит убыток в 347,87 тенге, а если банк увеличит на 1 тысячу тенге свои вложения в ценные бумаги, то банк потерпит убыток в 225,42 тенге. т.е. банку в это время нельзя заниматься традиционными операциями» [1].

Для иллюстрации статистического подхода к финансовому экспресс-анализу нужны модельные данные, адекватные по значениям статистик много мерной выборки. Перечень этих статистик (векторы, матрицы) будет выявлен по мере изложения текста. При этом закон распределения значений 1-мерных переменных для финансовых показателей бывает неопределенным, что достигается применением обратной модели главных компонент (ОМ ГК) [2,3], для 1-мерных z-переменных из R-, $\Lambda$ выборок не определены законы распределений.

\section{Модели и задачи}

Исходной гипотезой для рассматриваемой ниже обратной задачи множественной линейной регрессии (О3 МЛРА) является существование уравнения регрессии вида $\mathrm{z}_{\mathrm{n}}=\beta_{1} \mathrm{z}_{1}+\beta_{2} \mathrm{z}_{2}+\ldots+\beta_{\mathrm{n}-1} \mathrm{z}_{\mathrm{n}-}$ 1 , где, в отличие от прямой задачи множественной линейной регрессии (ПЗ МЛРА) известны значения $\beta_{1}, \ldots, \beta_{\mathrm{n}-1}$ вектора $\beta=\left(\beta_{1}, \ldots, \beta_{\mathrm{n}}\right.$ $\left.{ }_{1}\right)^{\mathrm{T}}$ регрессионных коэффициентов, значение свободного члена $\alpha$. Модель множественной линейной регрессии, где вычисляется единственный вектор $\quad \beta=\left(\beta_{1}, \ldots, \beta_{\mathrm{n}-1}\right)^{\mathrm{T}}$ регрессионных коэффициентов, назовем (при $\alpha=0)$ прямой моделью множественной линейной регрессии (ПМ МЛРА) и обозначим так: $Z_{\mathrm{mn}}=\left[\mathrm{Z}_{1} \mid \mathrm{Z}_{2}\right]=>\left(\mathrm{R}_{11}, \mathrm{R}_{12}, \beta\right)$. В ПМ МЛРА решена ПЗ МЛРА, ее решение $\beta$ единственно и равно $\beta_{\mathrm{R}}=\mathrm{R}^{-1}{ }_{11} \mathrm{R}_{12}$. Для каждого значения $\mathrm{Z}_{\mathrm{n}}$ из «реальной» выборки и значения $\mathrm{Z}_{\mathrm{n}}$ из ПММЛА разность величины остатка случайно не равно нулю. В нашей ОМ МЛРА аналогичная разность равна нулю. В ОМ МЛРА модельные значения $\mathrm{n}$ $\mathrm{z}$-переменных точно удовлетворяют $\mathrm{z}_{\mathrm{n}}=\beta_{1} \mathrm{Z}_{1}+\beta_{2} \mathrm{z}_{2}$ $+\ldots+\beta_{\mathrm{n}-1} Z_{\mathrm{n}-1}$. Аддитивное случайное приращение $\alpha_{i}, \mathrm{i}=1, \ldots, \mathrm{m}$, к значениям $\mathrm{z}_{\mathrm{in}}$ придает вектору-

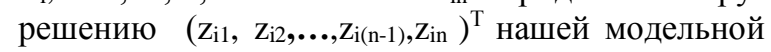
выборки соответствует ошибке предсказанного значения в ПМ МЛРА. Следовательно теоретическое решение ПМ МЛРА является одним из бесконечного множества теоретических решений ОМ МЛРА.

Моделируемые данные соответствуют данным из конвертированных балансов предприятия [3]. Обоснование соответствия наших модельных данных данным из конвертированных балансов проведены в разделе статьи [2]. Эта переоценка похожа на прогнозирование будущего (именно для этого чаще всего служит моделирование). И не есть восстановление прошлого, т.е. «обратное моделирование».

Трудным местом ПЗ МЛРА[1] является вычисление обратной матрицы для симметрической корреляционной матрицы «регрессоров» $\mathrm{R}_{11}$, которая может быть неполного ранга - тогда не существует для нее обратной матрицы, или быть «плохо обусловленной». Число обусловленности матрицы показывает насколько матрица близка к матрице неполного ранга (для квадратных матриц - к вырожденности). В работах [5-14] число обусловленности корреляционной матрицы измеряется набором еe f-параметров $\left.\mathrm{f}_{1}\left(\Lambda_{\mathrm{nn}}\right)=\lambda_{1}+\ldots+\lambda_{\mathrm{n}}\right)=\mathrm{n}, \mathrm{f}_{2}\left(\Lambda_{\mathrm{nn}}\right)=$ $\left(\lambda^{2}{ }_{1}+\ldots+\lambda^{2}{ }_{n}\right), f_{3}\left(\Lambda_{n n}\right)=\lambda_{1} / \lambda_{n}$, $\mathrm{f}_{4}\left(\Lambda_{\mathrm{nn}}\right)=\left(\lambda_{1}+\ldots+\lambda_{\ell}\right) / \mathrm{n}<1, \mathrm{f}_{5}\left(\Lambda_{\mathrm{nn}}\right)=\lambda_{1} \times \lambda_{2} \times \lambda_{3} \times \ldots \times \lambda_{\mathrm{n}}, \mathrm{f}_{6}($ $\left.\left.\Lambda_{\mathrm{nn}}\right)=\quad \lambda_{1} / \lambda_{2}+\ldots+\lambda_{\mathrm{n}-1} / \lambda_{\mathrm{n}}\right)$.Здесь $\mathrm{f}_{3}\left(\Lambda_{\mathrm{nn}}\right)=\lambda_{1} / \lambda_{\mathrm{n}}$ измеряяет значение числа обусловленности, а остальные - близость (удаленность) от вырожденности корреляционной матрицы $\mathrm{R}_{\mathrm{nn}}$. Но при этом решается прямая спектральная задача (ПСЗ): $\mathrm{R}_{\mathrm{nn}}=>\left(\mathrm{C}_{\mathrm{nn}}, \Lambda_{\mathrm{nn}}\right), \quad$ где квадратная ортонормированная матрица $\mathrm{C}_{\mathrm{nn}},-$ матрица собственных векторов $\mathbf{c}_{\mathbf{j}}=\left(\mathrm{c}_{1 \mathrm{j}}, \mathrm{c}_{2 \mathrm{j}} \ldots \mathrm{c}_{\mathrm{nj}}\right)^{\mathrm{T}}, \mathrm{j}=1, \ldots, \mathrm{n}$, образующих ортогональную матрицу $\mathrm{C}_{\mathrm{nn}}=\left[\mathbf{c}_{1}\left|\mathbf{c}_{2}\right| \ldots \mid \mathbf{c}_{\mathbf{n}}\right], \quad$ согласованную с матрицей собственных чисел (спектром) $\Lambda_{n n}=\operatorname{diag}\left(\lambda_{1}, \lambda_{2}, \ldots, \lambda_{n}\right), \lambda_{1}>\ldots>\lambda_{n}>0$, таким образом, что выполняются равенства $\mathrm{R}_{\mathrm{nn}} \mathrm{C}_{\mathrm{nn}}=\mathrm{C}_{\mathrm{nn}} \Lambda_{\mathrm{nn}}, \mathrm{C}^{\mathrm{T}}{ }_{\mathrm{nn}} \mathrm{C}_{\mathrm{nn}}=\mathrm{C}_{\mathrm{nn}} \mathrm{C}^{\mathrm{T}}{ }_{\mathrm{nn}}=\mathrm{I}_{\mathrm{nn}}, \quad$ где $\operatorname{diag}\left(\mathrm{R}_{\mathrm{nn}}\right)=(1, \ldots, 1)$,

$\operatorname{tr}\left(\mathrm{R}_{\mathrm{nn}}\right)=1+1+1+1+1+1=\operatorname{tr}\left(\Lambda_{\mathrm{nn}}\right)=\lambda_{1}+\ldots+\lambda_{\mathrm{n}}=\mathrm{n} \quad[4-11]$. Матрицы $\mathrm{C}_{\mathrm{nn}}$ и $\Lambda_{\mathrm{nn}}$ вычислятся одновременно по известной корреляционной матрице $\mathrm{R}_{\mathrm{nn}}$. Матрица $\mathrm{R}_{\mathrm{nn}}$ вычисляется по стандартизованной выборке $\mathrm{Z}_{\mathrm{mn}}: \quad \mathrm{R}_{\mathrm{nn}}=(1 / \mathrm{m}) \mathrm{Z}^{\mathrm{T}}{ }_{\mathrm{mn}} \mathrm{Z}_{\mathrm{mn}}$. Элементы спектра $\Lambda_{\mathrm{nn}}=\operatorname{diag}\left(\lambda_{1}, \ldots, \lambda_{\mathrm{n}}\right), \quad \mathrm{n}>2, \quad$ являются вышеприведенными измерителями.

Решаемая в [1] О3 МЛРА, как показано в «Теореме о z-переменных в ОМ МЛРА» [1], имеет бесконечное множество решений $\left(\mathrm{R}^{(\ell)}{ }_{11}, \mathrm{R}^{(\ell)}{ }_{12}, \quad \mathrm{Z}^{(\mathrm{t})}{ }_{1}, \mathrm{Z}^{(\ell, \mathrm{t})}{ }_{2}\right), \quad$ где $\quad$ матрицы корреляционные матрицы $\mathrm{R}^{(\ell)}{ }_{11}$ моделируются в модели вида: $\left(\mathrm{n}, \varphi_{11}\right)=>\left(\mathrm{R}^{(\ell)}{ }_{11}\right)$, подматрицы $\mathrm{R}^{(\ell)}{ }_{12}$ вычисляются: $\mathrm{R}^{(\ell)}{ }_{12}=\mathrm{R}^{(\ell)}{ }_{11} \beta$, подматрицы $\mathrm{Z}^{(\ell, \mathrm{t})}{ }_{1}$ являются решением О3 АГК: $\mathrm{R}^{(\ell)}{ }_{11}=>\left(\mathrm{C}^{(\ell)}{ }_{11}, \Lambda^{(\ell)}{ }_{11}, \mathrm{Y}^{(\mathrm{t})}{ }_{\mathrm{m}(\mathrm{n}-1)}, \mathrm{Z}^{(\ell, \mathrm{t})}{ }_{\mathrm{m}(\mathrm{n}-1)}\right)$, подматрица $Z^{(\ell, t)}{ }_{2}$-решением Оптимизационной задачи №5, $\mathrm{t}=1, \ldots \mathrm{K}_{\mathrm{t}}<\infty, \ell=1, \ldots, \mathrm{k}_{\ell}<\infty$. Выборки $Z^{(\mathrm{t})}{ }_{1}, Z^{(\ell, \mathrm{t})}{ }_{2} \mathrm{OM}$ ГК удовлетворяют соотношениям: $(1 \backslash \mathrm{m}) Z^{(\mathrm{t}) \mathrm{T}}{ }_{1} \mathrm{Z}^{(\mathrm{t})}{ }_{1}=\mathrm{R}^{(\ell)}{ }_{11},(1 \backslash \mathrm{m}) \mathrm{Z}^{(\mathrm{t}) \mathrm{T}}{ }_{1} \mathrm{Z}^{(\ell, \mathrm{t})}{ }_{2}=\mathrm{R}^{(\ell)}{ }_{12}$,

$(1 \backslash \mathrm{m}) Z^{(\ell, \mathrm{t}) \mathrm{T}}{ }_{2} Z^{(\ell, \mathrm{t})}{ }_{2}=\mathrm{R}_{22}=1$. Матрицы $\quad \mathrm{C}^{(\ell)}{ }_{11}$, $\left.\Lambda^{(\ell)}{ }_{11}, Y^{(\mathrm{t})}{ }_{\mathrm{m}(\mathrm{n}-1)}, \quad \mathrm{Z}^{(\ell, \mathrm{t})}{ }_{\mathrm{m}(\mathrm{n}-1)}\right) \quad$ из решаемых задач используются для достижения требуемых 
равенств, удовлетворяют соотношениям ОМ ГК, доказанным в Теореме о $\Lambda$-выборках $[5,7,9]$.

\section{Обратная модель множественной линейной регрессии}

ОМ МЛРА разработана в работе [1]. Она необходима для моделирования данных, демонстрирующих все возможные динамики в моменты времени $\mathrm{t}$ рассматриваемых нами агрегированных коэффициентов $\beta^{(t)}{ }_{R}=\left(\beta^{(t)} 1, \ldots, \beta^{(t)}{ }_{n}\right.$ 1). Здесь $\mathrm{t}$ означает момент времени даты бухгалтерского баланса, данные из которого (или первичные данные, трансформированные к моменту времени t) используются в нашей модели. Для коэффициентов $\beta^{(\mathrm{t})} \mathrm{R}=\left(\beta^{(\mathrm{t})} 1, \ldots, \beta^{(\mathrm{t})}{ }_{\mathrm{n}-1}\right)^{\mathrm{T}}$ существуют соответствующие подматрицы $\mathrm{R}^{(\ell)}{ }_{11}, \mathrm{R}^{(\ell)}{ }_{12}$, корреляционных матриц, подматрицы $\mathrm{Z}$-переменны $\mathrm{Z}_{1}, \ldots, \mathrm{Zn}-1_{\mathrm{n}}: \quad(1 \backslash \mathrm{m}) \mathrm{Z}^{(\mathrm{t}) \mathrm{T}}{ }_{1} \mathrm{Z}^{(\mathrm{t})}{ }_{1}=\mathrm{R}^{(\ell)}{ }_{11}$, $(1 \backslash \mathrm{m}) Z^{(\mathrm{t}) \mathrm{T}}{ }_{1} Z^{(\ell, \mathrm{t})}{ }_{2}=\mathrm{R}^{(\ell)}{ }_{12}$, на независимые- $\mathrm{Z}_{1}, \ldots, \mathrm{Z}_{2} \quad$ и зависимую- $\mathrm{Z}_{\mathrm{n}}=\beta_{1} \mathrm{Z}_{1}+, \ldots, \beta_{\mathrm{n}-1} \mathrm{Z}_{\mathrm{n}-1}, \quad \mathrm{R}^{(\ell)}{ }_{12}=\mathrm{R}^{(\ell)} \mathbf{1 1} \mathbf{\beta}$, показателей Динамики этих показателей показывают оптимистические или неблагоприятные тенденции в периоды времени t, наличие которых мы будем определять по коэффициентам корреляции, по коэффициентам эластичности прибыли по объясняющему фактору с номером j, где j может принимать одно из значений $1,2 \ldots, \mathrm{n}-1$.

Для значений коэффициентов регрессии (входных параметров модели) в статье [ 1] решены задача 1 , подзадача 1 , подзадача 2, подзадача 3, оптимизационная задача №5, доказана Теорема.

ОМ МЛРА [1]: $\boldsymbol{\beta}=>\left[\mathbf{Z}^{(\ell, t)} \mathbf{1} \mid \mathbf{Z}^{(\ell, t)} \mathbf{2}\right]$ существует, имеет бесконечное множество решений с номерами $\mathrm{t}=1, \ldots, \mathrm{K}_{\mathrm{t}}<\infty, \ell=1, \ldots, \mathrm{\kappa}_{\ell}<\infty$. Выходным объектом О3 МЛРА является многомерная выборка $\mathrm{Z}_{\mathrm{mn}}=\left[\mathrm{Z}_{1} \mid \mathrm{Z}_{2}\right]=\left\{\left(\mathrm{Z}_{\mathrm{i} 1}, \ldots, \mathrm{Z}_{\mathrm{i}, \mathrm{n}-1} \mid \mathrm{Z}_{\mathrm{in}}\right)\right\}$, Входным объектом обратной задачи множественной линейной регрессии (ОЗ МЛРА) является вектор $\beta_{\mathrm{R}}=\left(\beta_{1}, \ldots, \beta_{\mathrm{n}-1}\right)$. Выходным объектом О3 МЛРА является многомерная выборка $\mathrm{Z}_{\mathrm{mn}}=\left[\mathrm{Z}_{1} \mid \mathrm{Z}_{2}\right]=\{($ $\left.\left.\mathrm{Z}_{\mathrm{i} 1}, \ldots, \mathrm{Z}_{\mathrm{i}, \mathrm{n}-1} \mid \mathrm{Z}_{\mathrm{in}}\right)\right\}$.

На шаге 1 подзадачи 1 моделируются матрицы $\mathrm{R}^{(\ell)}{ }_{11}$ в модели вида: $\left(\mathrm{n}, \varphi_{11}\right)=>\left(\mathrm{R}^{(\ell)}{ }_{11}\right)$, подматрицы $\mathrm{R}^{(\ell)}{ }_{12}$ вычисляются: $\mathrm{R}^{(\ell)}{ }_{12}=\mathrm{R}^{(\ell)}{ }_{11} \beta$, подматрицы $Z^{(\ell, t)}{ }_{1}$ являются решением О3 АГК: $\mathrm{R}^{(\ell)}{ }_{11}=>\left(\mathrm{C}^{(\ell)}{ }_{11}, \Lambda^{(\ell)}{ }_{11}, \mathrm{Y}^{(\mathrm{t})}{ }_{\mathrm{m}(\mathrm{n}-1)}, \mathrm{Z}^{(\ell, \mathrm{t})} \mathrm{m}(\mathrm{n}-1)\right)$, подматрица $Z^{(\ell, t)} 2$-решением Оптимизационной задачи №5, $\mathrm{t}=1, \ldots, \mathrm{K}_{\mathrm{t}}<\infty, \ell=1, \ldots, \mathrm{k}_{\ell}<\infty$. Выборки $\mathrm{Z}^{(\mathrm{t})}{ }_{1}, \mathrm{Z}^{(\ell, \mathrm{t})}{ }_{2} \mathrm{OM}$ ГК удовлетворяют соотношенииям $(1 \backslash \mathrm{m}) Z^{(\ell, \mathrm{t}) \mathrm{T}}{ }_{1} \mathrm{Z}^{(\ell, \mathrm{t})}{ }_{1}=\mathrm{R}^{(\ell)}{ }_{11}, \quad(1 \backslash \mathrm{m}) \mathrm{Z}^{(\ell, \mathrm{t}) \mathrm{T}}{ }_{1} \mathrm{Z}^{(\ell, \mathrm{t})}=\mathrm{R}_{2}{ }^{(\ell)}{ }_{12}$, $(1 \backslash \mathrm{m}) \mathrm{Z}^{(\ell, \mathrm{t}) \mathrm{T}}{ }_{2} \mathrm{Z}^{(\ell, \mathrm{t})}{ }_{2}=\mathrm{R}_{22}=1$. При этом матрицы $\mathrm{C}^{(\ell)}{ }_{11}$, $\Lambda^{(\ell)}{ }_{11}, \mathrm{Y}^{(\mathrm{t})}{ }_{\mathrm{m}(\mathrm{n}-1)}, \mathrm{Z}^{(\ell, \mathrm{t})}{ }_{\mathrm{m}(\mathrm{n}-1)}$ из решаемых задач используются для достижения требуемых равенств, удовлетворяют соотношениям ОМ ГК, доказанным в Теореме о $\Lambda$-выборках [9].
Конкретные значения полученного решения $\mathrm{Z}_{1 \mathrm{j}}, \mathrm{Z}_{2 \mathrm{j}}, \ldots, \mathrm{Z}_{\mathrm{mj}}$ системы уравнениц (*) зависят от начальных значений, назначаемых пользователем процедуры «Поиск решения» и вводимых в соответствующее поле окна этой процедуры.

Ниже в примере мы выбрали любой нормированный вектор $\left(\mathrm{Z}_{1 \mathrm{j}}, \mathrm{Z}_{2 \mathrm{j}}, \ldots, \mathrm{Z}_{\mathrm{mj}}\right)$ такой, что $1=\left(\mathrm{z}_{1 \mathrm{j}}{ }^{2}+\ldots+\mathrm{z}_{\mathrm{m}, \mathrm{j}}{ }^{2}\right) / \mathrm{m}$, где $\mathrm{n}=3, \mathrm{~m}=20$. Этот вектор в паре с другими векторами из столбцов матрицы $\mathrm{Z}_{\mathrm{mn}}$ не дает желаемых значений коэффициентов корреляции, но процедура «Поиск решения» легко преобразует этот нормированный вектор $\left(\mathrm{z}_{1 \mathrm{j}}, \mathrm{Z}_{2 \mathrm{j}}, \ldots, \mathrm{Z}_{\mathrm{mj}}\right)$ в вектор, являющийся решением системы (*). Размерность $\mathrm{n}=3$ в нашем примере позволяет сравнивать результаты расчетов с данными, полученных в результате анализа данных из работ [5-14].

\section{Примеры многомерных МЛРА-выборок z-переменных, моделируемых в ОМ МЛРА}

Приведем две многомерные МЛРА-выборки z-переменных, моделируемых с применением ОМ МЛРА. Этот пример анализирует случай $\mathrm{n}=3$, удобен для восприятия особенностей ОМ МЛРА. Случай $\mathrm{n}=4$ сложен в этом аспекте. В примере реальными являются величины $\boldsymbol{\beta}_{1}=-$ 0.34787; $\boldsymbol{\beta}_{2}=-\mathbf{0 , 2 2 5 4 2}$., Значения выборочных средних х1 ${ }^{\text {pp= }=329.6}$ (себестоимость реализованной продукции (товаров, услуг), млн. тенге), $\mathbf{x}_{2}{ }^{\mathbf{c}}=\mathbf{8 4 1 . 3}$ (стоимость основных средств, финансовые инвестиции, фонд заработанной платы и другие капиталы, млн. тенге), $\mathbf{x}^{\mathbf{c p}}=\mathbf{4 5 6 . 9}$, (доход от реализации продукции (товаров и услуг), млн. тенге), стандартные отклонения $s_{1}=51,1749, s_{2}=109,3621, s_{3}=105,1742$.

В МЛРА-выборке $\quad Z_{\mathrm{mn}}=\left[\mathrm{Z}^{\left(\mathrm{t}, \ell,{ }_{1}\right.} \mid \mathrm{Z}^{(\mathrm{t}, \ell)}{ }_{2}\right]$ моделируются преобразованные многомерные случайности $\quad[5,7,12-14] \quad$ преобразуемые случайными преобразованиями от одномерных случайностей, генерируемых датчиком случайных чисел, равномерно распределенных в интервале $[0,1]$. Заметим, что реализовать наше случайное число из интервала $[0,1]$, имеющую вид бесконечной дроби в компьютере невозможно, так как разрядная сетка компьютера ограничена. В компьютере можно формировать дискретные последовательности случайных чисел, которые не могут отличаться друг от друга только на величину меньше $2^{\text {-n }}$ (n-число разрядов в ячейке компьютера, $\mathrm{n}=64)$. То есть непрерывного, "теоретического" распределения на компьютерах получить нельзя. Если эти числа равновероятны, то такое распределение случайных чисел называют квазиравномерным.

Этапы применения программ из ППП «Спектр» для получения многомерной $\Lambda$ выборки z-переменных, точно удовлетворяющих соотношениям, уравнениям ОМ МЛРА (O3 
МЛРА объединим и изобразим так: $\left(\mathbf{m}=\mathbf{2 0}, \mathbf{n}=\mathbf{2}, \mathbf{r}_{12}, \mathbf{B}=\left(\mathbf{B}_{1}, \mathbf{B}_{2}\right)^{\mathbf{T}}\right)=>\left(\mathbf{R}_{11}, \mathbf{R}_{12}, \quad \mathbf{Z}_{1}, \mathbf{Z}_{2}\right)$. состоят из следующих шагов. На Шаге 0 при $n=3$ моделируем спектр неизвестной корреляционной матрицы $\Lambda_{22}=\operatorname{diag}\left(\lambda_{1}, \lambda_{2}\right)$ размерности 2 . Это число равно числу объясняющих (независимых) переменных («регрессоров»), наличие такого разбиения z-переменных требует отдельного моделирования значений их матриц $\mathrm{Z}_{1}, \mathrm{Z}_{2}$. Сперва в соответствии с значением $\mathrm{n}=2$ и со значениями $\lambda_{1}, \lambda_{2}$ известных элементов спектра $\Lambda_{22}$ моделируем одну корреляционную матрицу $\mathrm{R}^{(\ell)}{ }_{11}$ с номером $\ell=1, \ldots, \kappa_{\ell}<\infty$, размерности 2-на-2. Характеристику этой кореляционной матрицы выразим в виде значения параметра $\varphi_{11}[9]$. Схему этого Шага 1 мы обозначили так: (n$\left.1, \mathrm{r}^{(\ell)}\right)=>\left\{\Lambda^{(\ell)}{ }_{22}=\operatorname{diag}\left(\lambda^{(\ell)}{ }_{1}, \lambda^{(\ell)}{ }_{2}\right)\right\}$. Значения $\lambda^{(\ell)}{ }_{1}, \lambda^{(\ell)}{ }_{2}$ приведены в [9]: $\lambda^{(\ell)}{ }_{1}=1+\mathrm{r}^{(\ell)}, \lambda^{(\ell)}{ }_{2}=1-\mathrm{r}^{(\ell)}$, при $\mathrm{r}^{(\ell)}>0$. Если $\mathrm{r}^{(\ell)}<0$, то $\lambda^{(\ell)}{ }_{1}=1-\mathrm{r},{ }^{(\ell)}, \lambda^{(\ell)}{ }_{2}=1+\mathrm{r}^{(\ell)}$, Этим мы гарантируем выполнение обязательного условия: $\lambda^{(\ell)}{ }_{1}>\lambda^{(\ell)}{ }_{2}$. Для моделирования m-на-2-матрицы (2мерной выборки) $Z_{1}$, являющейся частью нашей будущей выборки $Z_{\mathrm{m} 3}=\left[\mathrm{Z}_{1} \mid \mathrm{Z}_{2}\right]$, необходимо моделировать 2-мерные выборки $\mathrm{U}_{\mathrm{m} 2}$ и $\mathrm{Y}_{\mathrm{m} 2}$.

Для получения 2-мерной случайной стандартизированной выборки $\mathrm{U}_{\mathrm{m} 2}$, имеющей корреляционную матрицу $\mathrm{I}_{22}=\operatorname{diag}(1,1), \mathrm{m}>\mathrm{n}=2$, можно воспользоваться процедурой декорелирующего преобразования $[5,7,9,16,17]$ : $\mathrm{V}_{\mathrm{mn}}^{\circ}=>\mathrm{U}_{\mathrm{mn}}$, апробированного в [7-9] при многих значениях $\mathrm{m}>\mathrm{n}>2$. Здесь матрица $\mathrm{V}^{\circ}$ m состоит из $2-\mathrm{x}$ столбцов и $\mathrm{m}$ строк. Каждый элемент $\mathrm{v}_{\mathrm{ij}}$ этой марицы $\left\{\mathrm{v}_{\mathrm{ij}}^{\circ}\right\} \mathrm{i}=1, \ldots, \mathrm{m}, \mathrm{j}=1,2, \quad$ является значением теоретической случайной величины $\xi$, имеющей теоретическое равномерное распределение в интервале [0,1]: $\xi \sim \mathrm{P}_{[0,1]}$.

Из матрицы $\mathrm{V}^{\circ}$ mn значений случайных чисел мы должны получить те числа, которые нам нужны. Иначе говоря «чужие» числа мы должны преобразовать в «свои» числа. «Свои» числа, объединенные в таблицы чисел обладают требуемыми нам алгебраическими свойствами. Мы рассматриваем случай $\mathrm{n}=2$ из ОМ ГК. Нам придется использовать 2 столбца из уже готовой матрицы $U_{m n}, m=20>n>2$. Ниже мы не приводим таблицу $\mathrm{U}_{\mathrm{m} 2}$, взятой из матрицы $\mathrm{U}_{\mathrm{mn}}, \mathrm{m}=20>\mathrm{n}>2$, но приводим значения элементов матриц $Z_{\mathrm{mn}}=\left[\mathrm{Z}^{(\mathrm{t}, \ell,)_{1}} \mid \mathrm{Z}^{(\mathrm{t}, \ell)}{ }_{2}\right]$ при фиксированных значениях $\mathrm{t}, \ell$. Данное декоррелирующее преобразование в работах $[5,7,9,16]$ схематично обозначено так: $\mathrm{V}_{\mathrm{mn}}^{\circ}=>\mathrm{U}_{\mathrm{mn}}$ причем оно (только при $\mathrm{n}>2$ ) может быть реализовано для любой выборки значений случайной величины $\xi$ в виде матрицы данных $\mathrm{V}^{\mathrm{o}(\mathrm{t})}{ }_{\mathrm{mn}}$ ранга $\mathrm{n}>2$ с номером $\mathrm{t}: \mathrm{t}=1, \ldots, \mathrm{k}_{\mathrm{t}}<\infty$. Элементы $\mathrm{v}_{\mathrm{ij}}^{\circ}$ выборки $\mathrm{V}_{\mathrm{mn}}^{\circ}=\left\{\mathrm{v}_{\mathrm{ij}}^{\circ}\right\}$ являются реализациями 1-мерной случайной величины $\xi$. Закон распределения ее - равномерный $\left(\xi \sim \mathrm{P}_{[0,1]}\right)$ или гауссов $\left(\xi \sim \operatorname{Gau}(0, \Sigma), \quad \Sigma=\mathrm{I}_{\mathrm{nn}}\right)$. После вычисления средних арифметических $\mathrm{v}_{1}{ }^{\mathrm{me}} \ldots, \mathrm{v}_{\mathrm{n}}{ }^{\mathrm{me}}$ для каждой из $\mathrm{n}>2$ столбцов матрицы $\mathrm{V}^{\circ}{ }_{\mathrm{mn}}=\left\{\mathrm{v}_{\mathrm{ij}}^{\circ}\right\}$ вычисляется матрица $\mathrm{V}_{\mathrm{mn}}=\left\{\mathrm{v}_{\mathrm{ij}}^{\circ}-\mathrm{v}_{\mathrm{j}}^{\mathrm{me}}\right\}, \mathrm{i}=1, \ldots, \mathrm{m}$, $\mathrm{j}=1, \ldots, n$. Многомерная выборка $\mathrm{V}_{\mathrm{mn}}$ преобразуется в случайную стандартизированную выборку $U_{\mathrm{mn}}$ из множества $N_{s}\left(0, \mathrm{I}_{\mathrm{nn}}\right)$ [5,7,9,12-14] n-мерных выборок, имеющих выборочные средние, выборочные корреляционные матрицы, в точности равные $0_{1 \mathrm{n}}, \mathrm{I}_{\mathrm{nn}}$. Используемое при этом преобразование является случайным и зависящим от случайной выборки $\mathrm{V}_{\mathrm{mn}}$. Тогда исходное теоретическое распределение $\left(\xi \sim \mathrm{P}_{[0,1]} \quad\right.$ или $\left.\xi \sim \mathrm{Gau}(0, \Sigma), \quad \Sigma=\mathrm{I}_{\mathrm{nn}}\right) \quad$ после преобразования становится теоретически неопределенным. Ранг матрицы $\mathrm{U}_{\mathrm{m} 2} \quad \operatorname{rk}\left(\mathrm{U}_{\mathrm{m} 2}\right)=2$ такой, что: $(1 / \mathrm{m}) \mathrm{U}^{\mathrm{T}}{ }_{\mathrm{m} 2} \mathrm{U}_{\mathrm{m} 2}=\mathrm{I}_{22}$, Декоррелирующее преобразование реализуется на первом этапе построения ОМ ГК [5,7,9,12-17] при $\mathrm{n}>2$. Но при $\mathrm{n}=2$ оно не применимо. И мы вынуждены использовать любые 2 столбца из $\mathrm{U}_{\mathrm{mn}}, \mathrm{m}>\mathrm{n}>2, \mathrm{n}$ $\neq 2$ [9]. При этом декоррелирующем преобразовании применяется ПЗ АГК [4-5,7]. Для получения нашей 2-мерной выборки $\mathrm{U}_{\mathrm{mn}} \in \boldsymbol{N}_{s}\left(0, \mathrm{I}_{\mathrm{mn}}\right), \quad \mathrm{m}>\mathrm{n}=2$ нельзя применить декорелирующие преобразование к выборке $\mathrm{V}_{\mathrm{m} 2}$, ибо при n=2 программа, например, метода Якоби не работает. Символ $\boldsymbol{N}_{s}\left(0, \mathrm{I}_{\mathrm{nn}}\right)$ обозначает № множество», а не «генеральная совокупность». Оба эти объекта бесконечны.

Далее преобразуем выборку $\mathrm{U}^{(\mathrm{t})} \mathrm{m} 2$ в выборку $\mathrm{Y}^{(\mathrm{t})}{ }_{\mathrm{m} 2}=\mathrm{U}^{(\mathrm{t})}{ }_{\mathrm{m} 2} \Lambda^{(1 / 2)}{ }_{22}$, где значения элементов диагональной матрицы $\Lambda^{(1 / 2)} 22$ равны значенииям квадратных корней от элементов матрицы $\Lambda_{22}=\operatorname{diag}\left(\lambda_{1}, \lambda_{2}\right): \quad \lambda^{(1 / 2)}{ }_{1}=\operatorname{sqrt}(1+\mathrm{r}), \quad \lambda^{(1 / 2)}{ }_{2}=\operatorname{sqrt}(1-\mathrm{r})$. Полученная 2-мерная выборка $\mathrm{Y}^{(\mathrm{t})}{ }_{\mathrm{m} 2}$ содержит в 1-ом столбце 1-ую главную компоненту (упеременную из з $\mathrm{m}$ зачений): $\mathbf{y}^{(\mathrm{t})}{ }_{1}=\left(\mathrm{u}^{(\mathrm{t})}{ }_{11} \times \operatorname{sqrt}(1+\mathrm{r}), \ldots, \mathrm{u}^{(\mathrm{t})}{ }_{\mathrm{m} 1} \times \operatorname{sqrt}(1+\mathrm{r})\right)^{\mathrm{T}}, \quad$ во $\quad 2-\mathrm{om}$ столбце- 2-ую главную компоненту (упеременную из $\mathrm{m}$ значений $): \mathbf{y}^{(\mathrm{t})}{ }_{2}=\left(\mathrm{u}^{(\mathrm{t})}{ }_{12} \times \operatorname{sqrt}(1-\right.$ $\left.\mathrm{r}), \ldots, \mathrm{u}^{(\mathrm{t})}{ }_{\mathrm{m} 2} \times \operatorname{sqrt}(1-\mathrm{r})\right)^{\mathrm{T}}, \quad$ где $\quad \mathrm{u}^{(\mathrm{t})}{ }_{11}, \ldots, \mathrm{u}^{(\mathrm{t})} \mathrm{m} 1$ $\mathrm{u}^{(\mathrm{t})}{ }_{12}, \ldots, \mathrm{u}^{(\mathrm{t})} \mathrm{m} 2$ суть значения $\mathrm{u}$-переменных $\mathbf{u}^{(\mathrm{t})}{ }_{1}=\left(\mathrm{u}^{(\mathrm{t})}{ }_{11}, \ldots, \mathrm{u}^{(\mathrm{t})} \mathrm{m} 1\right)^{\mathrm{T}}, \mathbf{u}^{(\mathrm{t})}{ }_{2}=\left(\mathrm{u}^{(\mathrm{t})}{ }_{12}, \ldots, \mathrm{u}^{(\mathrm{t})} \mathrm{m} 2\right)^{\mathrm{T}}$,

полученных выше в результате моделирования при $\mathrm{n}>2$ матрицы $\mathrm{U}_{\mathrm{mn}}$. Но используем результат при $\mathrm{n}>2$ для случая $\mathrm{n}=2$. Выборка $\mathrm{Y}^{(\mathrm{t})}{ }_{\mathrm{m} 2}=\mathrm{U}^{(\mathrm{t})}{ }_{\mathrm{m} 2} \Lambda^{(1 / 2)}{ }_{22}$, представленная в виде матрицы главных компонент, удовлетворяет стандартному условию некоррелированности ее у-переменных: если $\mathbf{y}^{(\mathrm{t}) 2}{ }_{\mathrm{i} 1}=\left((1+\mathrm{r}) \times\left(\mathrm{u}^{(\mathrm{t}) 2}{ }_{\mathrm{i} 1}\right), \mathbf{y}^{(\mathrm{t}) 2}{ }_{\mathrm{i} 2}=((1-\right.$ $\left.\mathrm{r}) \times\left(\mathrm{u}^{(\mathrm{t}) 2}{ }_{\mathrm{i} 2}\right)\right), \mathrm{i}=1, \ldots, \mathrm{m}$, то выполняется равенство $(1 / \mathrm{m}) \mathrm{Y}^{(\mathrm{t}) \mathrm{T}}{ }_{\mathrm{m} 2} \mathrm{Y}^{(\mathrm{t})}{ }_{\mathrm{m} 2}=\operatorname{diag}((1+\mathrm{r}),(1-\mathrm{r})), \quad$ эквивалентное равенству $(1 / \mathrm{m}) \times \operatorname{diag}\left[(1+\mathrm{r}) \times\left(\mathrm{u}^{(\mathrm{t}) 2}{ }_{11},+\ldots+\mathrm{u}^{(\mathrm{t}) 2}{ }_{\mathrm{m} 1}\right),(1-\right.$ $\left.\mathrm{r}) \times\left(\mathrm{u}^{(\mathrm{t}) 2}{ }_{12}+\ldots+\mathrm{u}^{(\mathrm{t}) 2}{ }_{\mathrm{m} 2}\right)\right]=\operatorname{diag}[(1+\mathrm{r}),(1-\mathrm{r})]$, причем 2 m-мерных вектора значений у-переменных будучи взаимно перпендикулярными, имеют разные длины, равные $\lambda^{(\ell)}{ }_{1}=1 \pm /-\mathrm{r}^{(\ell)}, \lambda^{(\ell)}{ }_{2}=1(-/+) \mathrm{r}^{(\ell)}$ : $(1 / \mathrm{m}) \times \operatorname{diag}\left(\mathbf{y}^{(\mathrm{t}) 2} \cdot \mathbf{1}, \mathbf{y}^{(\mathrm{t}) 2} \cdot \mathbf{2}\right)=\operatorname{diag}\left(\left(1+\mathrm{r}^{(\ell)}\right),\left(1-\mathrm{r}^{(\ell)}\right)\right)$. Если $\mathrm{r}^{(\ell)}>0$, то $\lambda^{(\ell)}{ }_{1}=1+\mathrm{r}^{(\ell)}, \lambda^{(\ell)}{ }_{2}=1-\mathrm{r}^{(\ell)}$, если $\mathrm{r}^{(\ell)}<0$, то $\lambda^{(\ell)}{ }_{1}=1-\mathrm{r}^{(\ell)}, \quad \lambda^{(\ell)}{ }_{2}=1+\mathrm{r}^{(\ell)}, \quad$ должно выполняться 
обязательное условие $\lambda^{(\ell)}{ }_{1}>\lambda^{(\ell)}{ }_{2}$. Этим мы реализовали схему Шага 1: (n$\left.1, \mathrm{r}^{(\ell)}{ }_{12}\right)=>\left\{\Lambda^{(\ell)}{ }_{22}=\operatorname{diag}\left(\lambda^{(\ell)}{ }_{1}, \ldots, \lambda^{(\ell)}{ }_{22}\right)\right\}$. При $\mathrm{n}=3$ на Шаге 2 моделирование корреляционной матрицы $\mathrm{R}^{(\ell)} 22$ проводится с использованием только формул. При $\mathrm{n}-1=2$ для множества значений $\left\{\mathrm{r}^{(\ell)}\right\}=\{0.3,0.5,0.562299064,0.6,0.65,0.7,0.75,0.8,0.8$ $5,0.9,0.93,0.95\}$ приведены только по одному экземпляру выборок $\mathrm{U}^{(\mathrm{t})}{ }_{\mathrm{m} 2}, \quad \mathrm{Y}^{(\mathrm{t})} \mathrm{m} 2, \mathrm{Z}^{(\mathrm{t}, \ell)}{ }_{\mathrm{m} 2}$ [9].

Здесь мы будем использовать 12 корреляционных матриц $\mathrm{R}^{(\ell)}{ }_{22}, \quad \ell=1, \ldots, 12$, со своими спектрами $\Lambda^{(\ell)}{ }_{22}=\operatorname{diag}\left(\lambda^{(\ell)}{ }_{1}, \lambda^{(\ell)}{ }_{2}\right)$, элементы которой по абсолютным величинам удовлетворяют нас и соответствуют выбранному значению $\mathrm{r}^{(\ell)}$ в предыдущем Шаге 1. Она генерирует матрицу $\mathrm{R}^{(\ell)}{ }_{11} \quad(\ell=1$, Таблица 1 , Таблица 3).

При $\mathrm{n}=2$ для бесконечного множества корреляционных матриц размерности $2 \times 2 \mathrm{R}^{(\ell)}{ }_{22}$ таких, что что ее элементы удовлетворяют соотношениям: $\quad \operatorname{diag}\left(\mathrm{R}^{(\ell)}{ }_{22}\right)=\left(\mathrm{r}^{(\ell)}{ }_{11}, \mathrm{r}^{(\ell)}{ }_{22}\right)=(1,1)$, внедиагональные элементы положительны $1>\mathrm{r}_{21}{ }^{(\ell)}=\mathrm{r}^{(\ell)}{ }_{12}>0 \quad$ (отрицательны: $\left.-1 \leq \mathrm{r}^{(\ell)} \leq 0\right)$, а ее собственные числа равны $\lambda^{(\ell)}{ }_{1}=1+\mathrm{r}^{(\ell)}, \lambda^{(\ell)}{ }_{2}=1-\mathrm{r}^{(\ell)}$ $\left(\lambda^{(\ell)}{ }_{1}=1-\mathbf{r}^{(\ell)}, \lambda^{(\ell)}{ }_{2}=1+\mathrm{r}^{(\ell)}\right)$ существуют [9]:

a) только 2 собственных вектора c. $1=(-\sin \alpha, \cos \alpha)^{\mathrm{T}}, \quad \mathbf{c} .2=(\cos \alpha, \sin \alpha)^{\mathrm{T}}, \mathbf{c} \cdot 2=(\cos \alpha, \sin \alpha)^{\mathrm{T}}$, $\left(\mathbf{c} .1=(\sin \alpha, \cos \alpha)^{\mathrm{T}}, \quad \mathbf{c} \cdot 2=(\cos \alpha,-\sin \alpha)^{\mathrm{T}}\right), \quad$ такие, что $\mathbf{c}_{. \mathbf{i}}{ }^{\mathrm{T}} \mathbf{c}_{\mathbf{i}}=1, \mathbf{c}_{\mathbf{j}}{ }^{\mathrm{T}} \mathbf{c}_{\mathbf{j}}=1, \mathbf{c}_{\mathbf{i}}{ }_{\mathrm{T}}^{\mathrm{T}} \mathbf{c}_{\mathbf{j}}=0, \quad \mathrm{j}=1,2, \mathrm{i}=1,2, \quad \mathrm{i} \neq \mathrm{j}$. Это реализация ОМ ГК при $=2$, этого варианта не было в статьях, опубликованных ранее статьи.

Эти 2 матрицы 2 собственных векторов используем для моделирования подматрицы $Z_{1}$ (Таблица 1 , Таблица 3) из матрицы $\mathrm{Z}_{\mathrm{mn}}=\left[\mathrm{Z}_{1} \mid \mathrm{Z}_{2}\right]$. Она выбирается из числа выборок $Z_{1}=Z^{(\mathrm{t}, \ell)}{ }_{\mathrm{m} 2}$, $\mathrm{t}=1, \ldots, \mathrm{K}_{\mathrm{t}}<\infty, \ell=1,2$. Преобразуем полученную 2 мерную выборку $\mathrm{Y}^{(\mathrm{t})}{ }_{\mathrm{m} 2}=\mathrm{U}^{(\mathrm{t})}{ }_{\mathrm{m} 2} \Lambda^{(1 / 2)}{ }_{22}$ (объема $\mathrm{m}>2$ ) значений 2-х некоррелированных у-переменных в 2-мерную выборку значений 2-х коррелированных $\mathrm{Z}$-переменных $\mathrm{Z}_{2}=\mathrm{Z}^{(\mathrm{t}, \ell)}{ }_{\mathrm{m} 2}=\mathrm{Y}^{(\mathrm{t})}{ }_{\mathrm{m} 2} \mathrm{C}^{(\ell) \mathrm{T}}{ }_{22}$, где $\mathrm{i}$-ая строка матрицы $\mathrm{Z}_{\mathrm{m} 2}$ состоит из элементов

$$
\mathrm{z}^{(\mathrm{t}, \ell)} \quad \mathrm{i} 1=\left(\mathrm{u}_{\mathrm{i} 1} \times \operatorname{sqrt}(1+\mathrm{r}) \times(-\sin \alpha)+\mathrm{u}_{\mathrm{i} 2} \times \operatorname{sqrt}(1-\right.
$$

r) $\times \cos \alpha$,

$$
\mathrm{z}^{(\mathrm{t}, \ell)} \mathrm{i}_{2}=\left(\left(\mathrm{u}_{\mathrm{i} 1} \times \operatorname{sqrt}(1-\mathrm{r}) \times \cos \alpha-\mathrm{u}_{\mathrm{i} 2} \times \operatorname{sqrt}(1-\mathrm{r}) \times \sin \alpha\right)\right),
$$

a ее столбцы удовлетворяют условиям:

$$
\left.\left.\mathbf{z}^{(\mathrm{t}, \ell)} \cdot 1=\left(\mathrm{z}^{(\mathrm{t}, \ell}\right)_{11}, \ldots, \mathrm{z}^{(\mathrm{t}, \ell}\right)_{\mathrm{m} 1}\right)^{\mathrm{T}},
$$

$\mathbf{z}^{(\mathrm{t}, \ell)} \cdot \mathbf{2}=\left(\mathrm{z}^{(\mathrm{t}, \ell)}{ }_{1 \mathrm{j}}, \ldots, \mathrm{z}^{(\mathrm{t}, \ell)}{ }_{\mathrm{mj}}\right)^{\mathrm{T}}$,

$(1 / \mathrm{m}) \mathbf{z}^{(\mathrm{t}, \ell)} \cdot 1^{\mathrm{T}} \mathbf{z}^{(\mathrm{t}, \ell)} \cdot{ }_{2}=\mathrm{r}^{(\ell)}{ }_{12}=\mathrm{r}^{(\ell)}{ }_{21}=\mathrm{r}^{(\ell)}$,

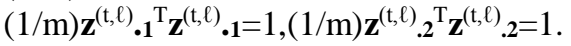

Далее вычисляем подматрицу $\mathrm{R}^{(\ell)}{ }_{12}=\mathrm{R}^{(\ell)}{ }_{11} \boldsymbol{\beta}$. Мы подошли к последнему шагу из их цепи: $\mathbf{B} \rightarrow \mathrm{R}^{(\ell)}{ }_{11} \rightarrow \mathrm{Z}^{(\ell, \mathrm{t})}{ }_{1} \rightarrow \mathrm{R}^{(\ell)}{ }_{12} \rightarrow \mathrm{Z}^{(\ell, \mathrm{t})}{ }_{2}$.

Здесь вектор коэффициентов регрессии $\boldsymbol{\beta}=\left(\boldsymbol{\beta}_{1}, \boldsymbol{B}_{2}\right)^{\mathbf{T}}$ задан нами и его компоненты равны $\boldsymbol{\beta}_{1}$ $=-\mathbf{0 . 3 4 7 8 7} ; \boldsymbol{\beta}_{2}=-\mathbf{0 , 2 2 5 4 2}$. Их значения (компонент вектора $\boldsymbol{\beta}$ и подматрицы $\mathrm{R}^{(\ell)}{ }_{12}$ ) приведены в Таблице1.
Теперь у нас имеются все данные для решения Оптимизационной задачи №5 (Рисунок 1, Таблица 1). Решение Оптимизационной задачи №5 схематично обозначим: $\left(\mathrm{Z}_{1}=\mathrm{Z}^{(\mathrm{t}, \ell)} \mathrm{m} 2, \mathrm{R}^{(\ell)}{ }_{12}\right)=>\mathrm{Z}_{2}=\mathrm{Z}^{(\ell, \mathrm{t})}{ }_{2} . \quad$ И смоделирована полная выборка $Z^{(t, \ell)}{ }_{m n}=\left[Z^{(t, \ell)}{ }_{1} \mid Z^{(t, \ell)}{ }_{2}\right]$, являющаяся решением нашей ОЗ МЛРА [1].

\section{Оптимизационная задача №5}

Пусть в модели многомерного линейного регрессионного анализа (МЛРА) задано разбиение множества 3 z-переменных на независимые - $\mathrm{z}_{1}, \ldots, \mathrm{Z}_{2}$ и зависимую - $\mathrm{z}_{3}$. По условию задачи существует уравнение регрессии вида $\mathrm{Z}_{3}=\beta_{1} \mathrm{Z}_{1}+\beta_{2} \mathrm{Z}_{2}$, где известны значения $\beta_{1}, \beta_{2}$ вектора $\beta=\left(\beta_{1}, \beta_{2}\right)^{\mathrm{T}}$ коэффициентов регрессии. В Шаге 1 решения подзадачи 2 задачи 1 [1] были получены значения элементов подматрицы $Z^{(\mathrm{t}, \ell)}{ }_{1}$. Эти значения будем использовать в качестве постоянных параметров уравнений в нижеприведенной системе (*).

Если известны значения 2 Z-переменных, расположенных в 2 столбцах подматрицы $Z_{1}$ размерности $\mathrm{m} \times 2$ и известны значения $\mathbf{r}_{13}, \mathbf{r}_{23}, \mathbf{r}_{33}$ 3-х коэффициентов корреляции (между 2 независимыми $\mathrm{z}_{1}, \ldots, \mathrm{z}_{2}$ и одной зависимой $\mathrm{z}_{3} \mathrm{Z}$ переменными) из подматрицы $\mathrm{R}_{12}=(1 / \mathrm{m}) \mathrm{Z}^{\mathrm{T}}{ }_{1} \mathrm{Z}_{2}$, то имеем систему из n-1 линейных уравнений и из 1 нелинейного уравнения:

$(1 / \mathrm{m}) \times\left(\mathrm{z}_{11} \times \mathrm{z}_{13}+\mathrm{Z}_{21} \times \mathrm{Z}_{23}+\ldots+\mathrm{Z}_{\mathrm{k} 1} \times \mathrm{Z}_{\mathrm{k} 3}+\ldots+\mathrm{z}_{\mathrm{m} 1} \times \mathrm{Z}_{\mathrm{m} 3}\right)=\mathbf{r}_{13}$ $(1 / \mathrm{m}) \times\left(\mathrm{z}_{21} \times \mathrm{z}_{13}+\mathrm{z}_{22} \times \mathrm{z}_{23}+\ldots+\mathrm{z}_{\mathrm{K} 2} \times \mathrm{z}_{\mathrm{K} 3}+\ldots+\mathrm{z}_{\mathrm{m} 2} \times \mathrm{z}_{\mathrm{m} 3}\right)=\mathbf{r}_{23}$ $(1 / \mathrm{m}) \times\left(\mathrm{z}_{13} \times \mathrm{z}_{13}+\mathrm{z}_{23} \times \mathrm{z}_{23}+\ldots+\mathrm{z}_{\mathrm{k} 3} \times \mathrm{Z}_{\mathrm{k} 3}+\ldots+\mathrm{z}_{\mathrm{m} 3} \times \mathrm{z}_{\mathrm{m} 3}\right)=\mathbf{r}_{33}$

Требуется найти m-мерное решение-вектор $\mathrm{Z}_{2}=\left(\mathrm{z}_{13}, \mathrm{Z}_{23}, \ldots, \mathrm{Z}_{\mathrm{m} 3}\right)^{\mathrm{T}}$ из матрицы $\mathrm{Z}_{20,3}=\left[\mathrm{Z}_{1} \mid \mathrm{Z}_{2}\right]$, удовлетворяющее системе уравнений $(*)$.

Описание примера расчетов при $\mathrm{n}=3$ моделирования многомерной $\Lambda$-выборки zпеременных в ОМ МЛРА следующее. Из равенства $\mathrm{R}^{(\ell)}{ }_{12}=\mathrm{R}^{(\ell)}{ }_{11} \boldsymbol{\beta}$ имеем значения величин из правой части системы уравнений $(*)$ : $\mathrm{r}_{13}=$ $0.397941, \quad \mathrm{r}_{23}=-0.06427, \quad \mathrm{r}_{13}=1$ (Таблица 1). Значения 40 коэффициен тов $\mathrm{Z}_{11}, \mathrm{Z}_{21}, \ldots, \mathrm{Z}_{\mathrm{\kappa} 1}, \ldots, \mathrm{Z}_{20,1}$, $\mathrm{Z}_{21}, \mathrm{Z}_{22}, \ldots, \mathrm{Z}_{\mathrm{K}, 2}, \ldots, \mathrm{Z}_{20,2} \quad$ в 2 -х уравнениях с 20 неизвестными $\mathrm{Z}_{13}, \mathrm{Z}_{23}, \ldots, \mathrm{Z}_{20,3}$ из левой части системы уравнений (*) представлены в первых двух столбцах Таблицы 2. Решением системы уравнений (*) является вектор-столбец $\mathrm{Z}_{2}=\left(\mathrm{Z}_{13}, \mathrm{Z}_{23}, \ldots, \mathrm{Z}_{20,3}\right)^{\mathrm{T}}$, его элементы расположены в столбце №3 Таблицы 2.

Для решения данной системы трех уравнений разработана программа-таблица в ЭТ Excel-2003 с применением процедуры Solver (надстройка «Поиск решения»). В окне (Рисунок 2) надстройки «Поиск решения» в 2 ячейках одного столбца на листе ЭТ Excel поместим 3 
формулы левых частей уравнений из системы (*). В правом столбце от этого столбца в его ячейках введем значения (числа) коэффициентов $\left(\mathbf{r}_{13}, \mathbf{r}_{23}, \mathbf{r}_{33}\right)=(0.397941,-0.06427,1)$ Первую ячейку с формулами (2-ая из 3-х ячеек в 1-ой строке (11 $1,00)$ на Рисунке 1) назначим целевой. В строках №2 и №3 ниже целевой ячейки с формулой разместим формулы 2-х функций ограничений для $\mathrm{r}_{13}$ и $\mathrm{r}_{23}$. эти 3 формулы на Рисунке 1 присутствуют дважды: в комментарии («Начальные данные») и в тексте программы («Полученное решение»).

В разработанной программе-таблице (Рисунок 1) вводим в ее ячейки значения элементов системы линейных и одного нелинейного уравнений. Решаем эту систему методом Ньютона, применяя надстройку «Поиск решения» в ЭТ Excel. Параметры имеют вид, приведенный на Рисунке 2. Нажав на кнопку «Выполнить» найдем элементы подматрицы $\mathrm{Z}_{2}$, зависящую от выборки $Z_{1}$ и от матрицы $\mathrm{R}_{12}$ (столбец №3, Таблица 1). Элементы подматрицы $\mathrm{Z}_{2}$ являются значениями $\mathrm{z}$-переменной №3 (Таблица 2, столбец 3; Таблица 4, столбец 1).

Многомерная МЛРА-выборка $Z_{20,3}=\left[Z_{1} \mid Z_{2}\right] 3$ $\mathrm{x}$ Z-переменных, полученная нами, имеет вид, приведенный в Таблице 2.

Выборочные средние $\mathrm{x}_{1}{ }^{\mathrm{cp}}=329.6$ (себестоимость реализованной продукции (товаров, услуг), млн. тенге), $\mathrm{x}_{2}{ }^{\mathrm{cp}}=841.3$ (стоимость основных средств, финансовые инвестиции, фонд заработанной платы и другие капиталы, млн. тенге), х $_{3}{ }^{\text {cp }}=456.9$, (доход от реализации продукции (товаров и услуг), млн. тенге) были вычислены по реальным данным 20ти малых предприятий одного из районов Алматинской области. Их стандартные

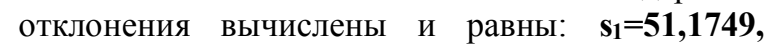
$\mathbf{s}_{2}=\mathbf{1 0 9 , 3 6 2 1 ,} \quad \mathbf{s}_{3}=\mathbf{1 0 5 , 1 7 4 2 .} \quad$ Стандартные отклонения имеют имя размерности «млн. тенге», совпадающее с именем размерности средних $\mathrm{x}_{1}{ }^{\mathrm{cp}}=329.6, \mathrm{x}_{2}{ }^{\mathrm{cp}}=841.3 ., \mathrm{x}_{3}{ }^{\mathrm{cp}}=456,9$. Они вычислены по 20 значениям центрированных х-переменных $\mathrm{x}_{1}, \mathrm{x}_{2}, \mathrm{x}_{3}$, где их значения удовлетворяют формуле $\mathrm{x}_{\mathrm{ij}}=\mathrm{x}_{\mathrm{ij}} \mathrm{x}^{\mathrm{cp}} \mathrm{j}_{\mathrm{j}} \mathrm{i}=1, \ldots, 20, \mathrm{j}=1,2,3$. На рисунке 4 приведены динамики изменений значений $3-\mathrm{x}$ zпеременных из МЛРА-выборки $Z^{(\mathrm{t}, \ell,)}{ }_{\mathrm{mn}}=\left[Z^{\left(\mathrm{t}, \ell,{ }_{1}\right.} \mid Z^{(\mathrm{t}, \ell)}{ }_{2}\right] . \quad$ МЛРА-выборка $Z^{(\mathrm{t}, \ell,)}{ }_{\mathrm{mn}}, \mathrm{m}=20$, удовлетворяет всем равенствам $(1 \backslash \mathrm{m}) \mathrm{Z}^{(\mathrm{t}) \mathrm{T}}{ }_{1} \mathrm{Z}^{(\mathrm{t})}{ }_{1}=\mathrm{R}^{(\ell)}{ }_{11}, \quad(1 \backslash \mathrm{m}) \mathrm{Z}^{(\mathrm{t}) \mathrm{T}}{ }_{1} \mathrm{Z}^{(\ell, \mathrm{t})}{ }_{2}=\mathrm{R}^{(\ell)}{ }_{12} \quad \mathrm{OM}$ МЛРА.

Мы привели описание шагов вычислений МЛРА-выборки при $\boldsymbol{\beta}_{\mathbf{1}}=\mathbf{0 , 6 3 4 7 8 7 ;} \boldsymbol{\beta}_{\mathbf{2}}=\mathbf{- 0 , 4 2 1 2 1}$.

Отрицательное значение $\boldsymbol{\beta}_{2}=\mathbf{- 0 , 4 2 1 2 1}$ обусловлено реальными факторами. Показатель «стоимость основных средств, финансовые инвестиции, фонд заработанной платы» отражает своим значением недавнее время своего воздействия как на показатель №1, так и на показатель №2. С течением времени показатель №2 должен дать положительное приращение показателю №3. Но он дает пока отрицательное приращение: $\quad \mathrm{z}_{3}=\left(\mathrm{z}_{2}+1\right)=\beta_{1} \mathrm{z}_{1}+\beta_{2}\left(\mathrm{z}_{2}+1\right)=$ $0.634787 \mathrm{z}_{1}+(-0.42121) \mathrm{z}_{2}=\mathrm{z}_{3}\left(\mathrm{z}_{2}\right)+(-0.42121)<\mathrm{z}_{3}\left(\mathrm{z}_{2}\right)$. Здесь $\mathrm{Z}_{3}\left(\mathrm{z}_{2}\right)$ означает функцию $\mathrm{z}_{3}$ от аргумента $\mathrm{z}_{2}$.

Проанализировав значения 3 -х $\mathrm{x}^{0}$ переменных, преобразованных из 3-х zпеременных, моделируемых в ОМ МЛРА, эксперты поставили задачу моделирования другой МЛРА-выборки с заданными значениями коэффициентов регрессии $\boldsymbol{\beta}_{1}=\mathbf{0 . 8 2 9 2 0}$; $\boldsymbol{\beta}_{2}=\mathbf{0 . 7 0 2 6 8 7 , ~ д а ю щ и х ~ п о л о ж и т е л ь н о е ~}$ приращение показателю №3 при приращениях на 1 как показателя №1, так и показателя №2. Для этого есть обоснование. Так как $\mathrm{Z}_{3}=\left(\mathrm{z}_{1}+1\right)=\mathrm{z}_{3}\left(\mathrm{z}_{1}\right)+(0.634787)>\mathrm{z}_{3}\left(\mathrm{z}_{1}\right) \quad$ и $\mathrm{z}_{3}=\left(\mathrm{z}_{2}+1\right)=\mathrm{z}_{3}\left(\mathrm{z}_{2}\right)+(-0.42121)<\mathrm{z}_{3}\left(\mathrm{z}_{2}\right)$, то $\mathrm{z}_{3}=\left(\mathrm{z}_{1}+1\right)-$ $\mathrm{z}_{3}\left(\mathrm{z}_{2}+1\right)=\left[\mathrm{z}_{3}\left(\mathrm{z}_{1}\right)-\mathrm{z}_{3}\left(\mathrm{z}_{2}\right)\right]+[0.634787+0.42121]$.

Это равенство показывает, что разность ( $\boldsymbol{\beta}_{1}$ $\boldsymbol{\beta}_{2}$ ) «с большой вероятностью» положительна: $\left(\boldsymbol{\beta}_{1}-\boldsymbol{\beta}_{\mathbf{2}}\right)>\mathbf{0}$. Неравенство $\boldsymbol{\beta}_{1}>\boldsymbol{\beta}_{2}$ у нас выполняется $\boldsymbol{\beta}_{\mathbf{1}}=\mathbf{0 . 6 3 4 7 8 7}>\boldsymbol{\beta}_{\mathbf{2}}=\mathbf{- 0 . 4 2 1 2 1}$. Необходимо модельно (перейти от убыточных данных к данным, моделирующим посредством уравнения регрессии положительный доход) увеличить значение коэффициента регрессии $\boldsymbol{\beta}_{2}$ до желаемого положительного значения. Увеличение его значения было проделано «короткими» шагами и за десяток шагов достигнуто значения $\boldsymbol{\beta}_{\mathbf{2}}=\mathbf{0 . 7 0 2 6 8 7}$. При этом из-за неизменности коэффициента корреляции $\mathrm{r}_{12}$ $=0,56229$ значение коэффициента регрессии $\beta_{1}$ также за десяток шагов увеличено до значения $\boldsymbol{\beta}_{\mathbf{1}}=\mathbf{0 . 8 2 9 2 0}$. В итоге значения коэффициентов регрессии зафиксированы на уровне $\boldsymbol{\beta}_{\mathbf{1}}=\mathbf{0 . 8 2 9 2 0}>\boldsymbol{\beta}_{\mathbf{2}}=\mathbf{0 . 7 0 2 6 8 7}$. Динамика изменений значений Аналогично решив задачу №5 (Программа-таблица для надстройки «Поиск решения» (Solver - Рисунок 5; Модельные значения элементов подматриц коэффициентов корреляции $\mathrm{R}^{(\ell)}{ }_{11}, \mathrm{R}^{(\ell)}{ }_{12}$, модельные значения коэффициентов регрессии $\boldsymbol{\beta}_{\mathbf{1}}=\mathbf{0 . 8 2 9 2 0}>\boldsymbol{\beta}_{\mathbf{2}}=\mathbf{0 . 7 0 2 6 8 7}-$ Таблица 3) найдем значения z-переменной №3 (Таблица 4, столбец 2).

Найдем элементы подматрицы $Z_{2}$, зависящую от выборки $\mathrm{Z}_{1}$ и от матрицы $\mathrm{R}_{12}$ (столбец №3, Таблица 1). Элементы подматрицы $\mathrm{Z}_{2}$ являются значениями №3 (Таблица 2, столбец 3; Таблица 4, столбец 1).

При моделировании МЛРА-выборки с заданными коэффициентами регрессии $\boldsymbol{\beta}_{1}=\mathbf{0 . 8 2 9 2 0 ;} \boldsymbol{\beta}_{2}=\mathbf{0 . 7 0 2 6 8 7}$ мы используем (при преобразовании в $\mathrm{x}^{0}$-переменные) те же значения средних и стандартных отклонений: $\mathbf{x}^{\mathbf{c p}}=\mathbf{3 2 9 . 6}$, $\mathrm{x}_{2}{ }^{\mathrm{cp}}=841.3, \quad \mathrm{x}_{3}{ }^{\mathrm{cp}}=456.9, \quad \mathrm{~s}_{1}=51,1749, \quad \mathrm{~s}_{2}=109,3621$,

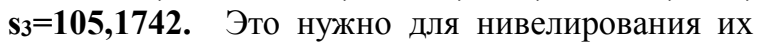
влияний при визуальном сравнении динамик 


\begin{tabular}{|c|c|c|c|c|c|c|}
\hline Impact Factor: & $\begin{array}{l}\text { ISRA (India) } \\
\text { ISI (Dubai, UAB } \\
\text { GIF (Australia) } \\
\text { JIF }\end{array}$ & $\begin{array}{l}=1.344 \\
=0.829 \\
=0.564 \\
=1.500\end{array}$ & $\begin{array}{l}\text { SIS (USA) } \\
\text { PИНЦ (Russia) } \\
\text { ESJI (KZ) } \\
\text { SJIF (Morocco) }\end{array}$ & $\begin{array}{l}=0.912 \\
=0.156 \\
=4.102 \\
=\mathbf{5 . 6 6 7}\end{array}$ & $\begin{array}{l}\text { ICV (Poland) } \\
\text { PIF (India) } \\
\text { IBI (India) }\end{array}$ & $\begin{array}{l}=6.630 \\
=1.940 \\
=4.260\end{array}$ \\
\hline
\end{tabular}

значений $\mathrm{x}^{0}$-переменных (Рисунок 7). Динамики изменений значений 3 -х $\mathrm{x}^{0}$-переменных, преобразованных из 3-x z-переменных по формуле $\mathrm{x}_{\mathrm{ij}}^{0}=\mathrm{Z}_{\mathrm{ij}} \mathrm{S}_{\mathrm{j}}+\mathrm{X}^{\mathrm{cp}}{ }_{\mathrm{j}}$ приведены на Рисунке 7 .

Описание расчетов (при $\boldsymbol{\beta}_{1}=\mathbf{0 . 8 2 9 2 0 ;} \boldsymbol{\beta}_{\mathbf{2}}=\mathbf{0 . 7 0 2 6 8 7}$ ) моделирования при новой многомерной МЛРА-выборки $3-\mathrm{x} \quad \mathrm{z}-$ переменных аналогично вышеприведенному описанию. Приведем только результаты. На Рисунке 5 программа-таблица для надстройки
«Поиск решения» (Solver), В Таблице 3 приведены модельные значения элементов подматриц коэффициентов корреляции $\mathrm{R}^{(\ell)}{ }_{11}$, $\mathrm{R}^{(\ell)}{ }_{12}$, реальные значения коэффициентов регрессии $\boldsymbol{\beta}_{\mathbf{1}}=\mathbf{0 . 8 2 9 2 0} ; \boldsymbol{\beta}_{\mathbf{2}}=\mathbf{0 . 7 0 2 6 8 7}$. В Таблице 4 , столбец №2 приведены значения новой $\mathrm{z}-$ переменной №3. Совместный график динамик двух решений задачи №5 - z-переменной №3 (Таблица 4), приведен на Рисунке 6.

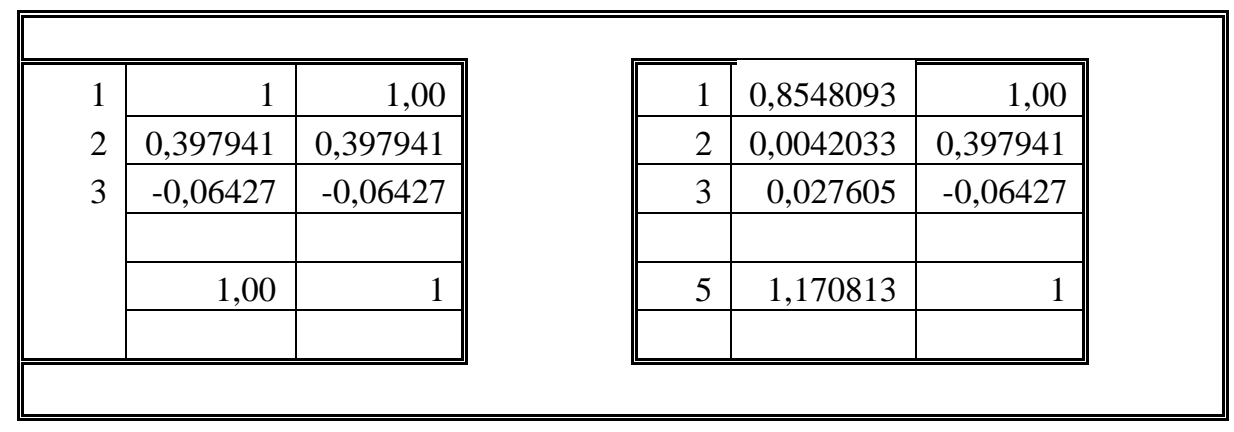

Рисунок 1 - Программа-таблица для надстройки «Поиск решения» (Solver)

Таблица 1

Модельные значения элементов подматриц коэффициентов корреляции $\mathbf{R}^{(\ell)}{ }_{11}, \mathbf{R}^{(\ell)}$, реальные значения коэффициентов регрессии $B_{3}=\left(\boldsymbol{B}_{1}, \boldsymbol{B}_{2}\right)^{T}$

\begin{tabular}{||c|c|c||c||}
\hline \multicolumn{2}{|c|}{$\mathrm{R}^{(\ell)}{ }_{11}$} & \multicolumn{1}{|c||}{$\mathrm{R}^{(\ell)} 12$} & $\mathbf{B}$ \\
\hline $\mathbf{1 , 0 0 0 0}$ & $\mathbf{0 , 5 6 2 2 9 9 0 6 4}$ & 0,397941 & 0,634787 \\
\hline $\mathbf{0 , 5 6 2 2 9 9 0 6 4}$ & $\mathbf{1 , 0 0 0 0}$ & $-0,06427$ & $-0,42121$ \\
\hline 0,397941 & $-0,06427$ & 1,0000 & \\
\hline
\end{tabular}

Таблица 2

МЛРА-выборка $Z_{m n}=\left[Z^{(t, \ell),} \mid Z^{(t, \ell)}{ }_{2}\right]$, удовлетворяющие равенствам $(1 \backslash \mathrm{m}) Z^{(t) \mathrm{T}}{ }_{1} Z^{(\mathrm{t})}{ }_{1}=R^{(\ell)}{ }_{11},(1 \backslash \mathrm{m}) Z^{(\mathrm{t}) \mathrm{T}}{ }_{1} Z^{(\ell, t)}{ }_{2}=R^{(\ell)}{ }_{12}, R^{(\ell)}{ }_{12}=R^{(\ell)}{ }_{11} \beta, \beta=(-0,347870,-0,225420)^{\mathrm{T}}$

\begin{tabular}{||c|c|c|c||}
\hline & \multicolumn{3}{|c|}{ Values of the z-variables $\mathbf{z}_{1}, \mathbf{z}_{2}, \mathbf{z}_{3}$} \\
\hline 0 & 1 & 2 & 3 \\
\hline 1 & $-0,26949$ & $-0,6751279$ & $-1,395987$ \\
\hline 2 & 0,851299 & 1,690445511 & 0,726718 \\
\hline 3 & 0,171842 & $-0,65191022$ & $-0,179229$ \\
\hline 4 & $-0,415423$ & 0,834632982 & $-1,05001$ \\
\hline 5 & $-1,061171$ & 0,174885282 & $-1,067333$ \\
\hline 6 & $-0,55355$ & $-0,65730942$ & $-0,521972$ \\
\hline 7 & $-1,635462$ & $-2,61243959$ & 0,64514 \\
\hline 8 & $-0,187172$ & $-0,37684687$ & 0,312487 \\
\hline 9 & 0,22706 & 0,495406099 & $-2,440673$ \\
\hline 10 & 1,417322 & 0,346405376 & 1,841887 \\
\hline 11 & $-0,253407$ & $-1,34115615$ & $-0,228297$ \\
\hline \multicolumn{4}{|c}{} \\
\hline
\end{tabular}




\begin{tabular}{|c|c|c|c|c|c|c|}
\hline Impact Factor: & $\begin{array}{l}\text { ISRA (India) } \\
\text { ISI (Dubai, UAE } \\
\text { GIF (Australia) } \\
\text { JIF }\end{array}$ & $\begin{array}{l}=1.344 \\
=0.829 \\
=0.564 \\
=1.500\end{array}$ & $\begin{array}{l}\text { SIS (USA) } \\
\text { PИНЦ (Russia) } \\
\text { ESJI (KZ) } \\
\text { SJIF (Morocco) }\end{array}$ & $\begin{array}{l}=0.912 \\
=0.156 \\
=4.102 \\
=\mathbf{5 . 6 6 7}\end{array}$ & $\begin{array}{l}\text { ICV (Poland) } \\
\text { PIF (India) } \\
\text { IBI (India) }\end{array}$ & $\begin{array}{l}=6.630 \\
=1.940 \\
=4.260\end{array}$ \\
\hline
\end{tabular}

\begin{tabular}{||c|c|c|c||}
12 & $-0,781238$ & $-0,87248111$ & $-0,337884$ \\
\hline 13 & $-0,920703$ & 1,258501727 & $-1,956155$ \\
\hline 14 & 1,708209 & 1,418344863 & 0,833235 \\
\hline 15 & $-1,281626$ & $-0,67603777$ & 0,11783 \\
\hline 16 & 0,765646 & 0,170802218 & 0,513218 \\
\hline 17 & 0,325761 & 0,432509081 & 0,230109 \\
\hline 18 & 0,226954 & $-0,241941$ & $-0,245842$ \\
\hline 19 & 2,346661 & 0,813930716 & 0,123245 \\
\hline 20 & $-0,681514$ & 0,469383841 & $-0,143386$ \\
\hline & 1,0000 & 1,0000 & 1,0000 \\
\hline
\end{tabular}

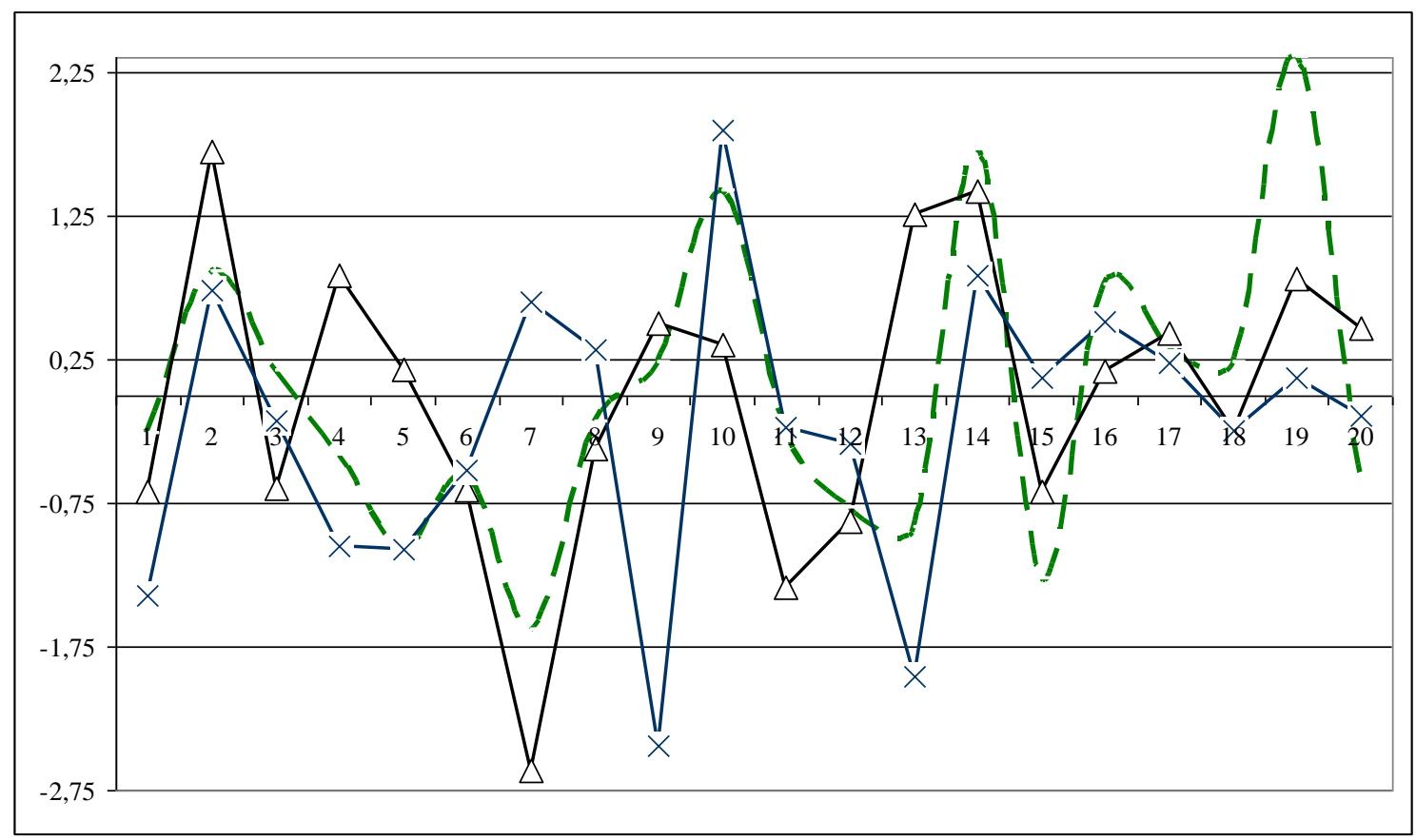

Рисунок 2 Динамики значений 3-x z-переменных, моделируемых в ОМ МЛРА $\beta_{1}=0,634787 ; \beta_{2}=-0,42121$

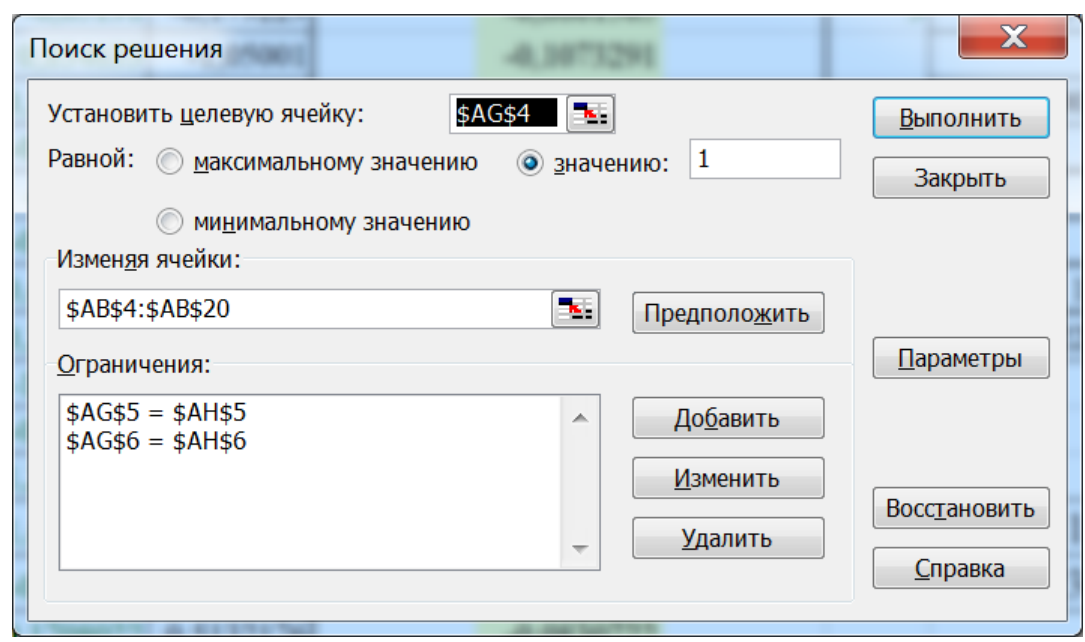

Рисунок 3 Окно процедуры «Поиск решений» в ЭТ Ехсеl с введенными формулами целевой функции оптимизационной задачи №5 $(n=3)$ и 2-х функций ограничений 


\begin{tabular}{|c|c|c|c|c|c|c|}
\hline Impact Factor: & $\begin{array}{l}\text { ISRA (India) } \\
\text { ISI (Dubai, UAE } \\
\text { GIF (Australia) } \\
\text { JIF }\end{array}$ & $\begin{array}{l}=1.344 \\
=0.829 \\
=0.564 \\
=1.500\end{array}$ & $\begin{array}{l}\text { SIS (USA) } \\
\text { PИНЦ (Russia) } \\
\text { ESJI (KZ) } \\
\text { SJIF (Morocco) }\end{array}$ & $\begin{array}{l}=0.912 \\
=0.156 \\
=4.102 \\
=\mathbf{5 . 6 6 7}\end{array}$ & $\begin{array}{l}\text { ICV (Poland) } \\
\text { PIF (India) } \\
\text { IBI (India) }\end{array}$ & $\begin{array}{l}=6.630 \\
=1.940 \\
=4.260\end{array}$ \\
\hline
\end{tabular}

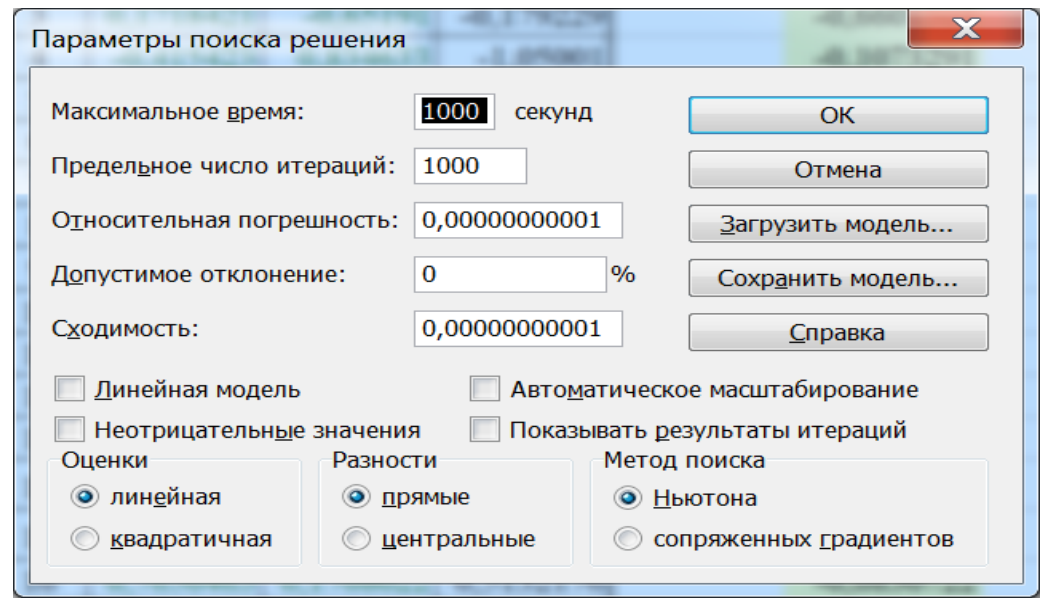

Рисунок 4 Окно «Параметры поиска решений» процедуры «Поиск решений» в ЭТ Ехсеl с введенными значениями параметров целевой функции оптимизационной задачи №5 $(n=3)$ и 3 функций ограничений

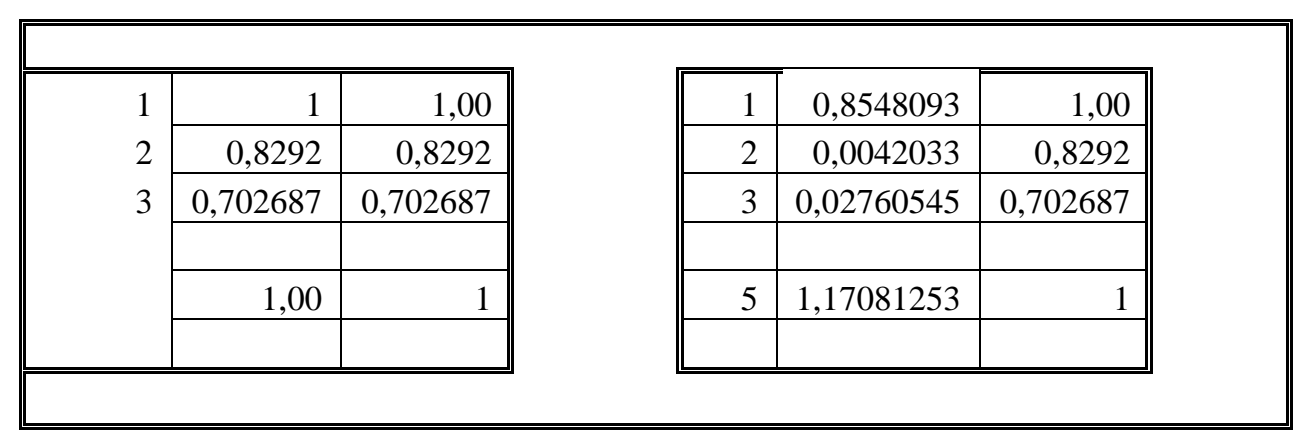

Рисунок 5 Программа-таблица для надстройки «Поиск решения» (Solver)

Таблица 3

Модельные значения элементов подматриц коэффициентов корреляции $\mathbf{R}^{(\ell)} 11, \mathbf{R}^{(\ell)}{ }_{12}$, модельные значения коэффициентов регрессии $\boldsymbol{B}_{3}=\left(\mathbf{B}_{1}, \mathbf{B}_{2}\right)^{T}$

\begin{tabular}{||c|c|c||c||}
\hline \multicolumn{2}{|c|}{$\mathrm{R}^{(\ell)}{ }_{11}$} & \multicolumn{1}{|c||}{$\mathrm{R}^{(\ell)}{ }_{12}$} & \multicolumn{1}{|c|}{$\mathbf{3}$} \\
\hline $\mathbf{1 , 0 0 0 0}$ & $\mathbf{0 , 5 6 2 2 9 9 0 6 4}$ & 0.634787 & $\mathbf{0 , 8 2 9 2 0}$ \\
\hline $\mathbf{0 , 5 6 2 2 9 9 0 6 4}$ & $\mathbf{1 , 0 0 0 0}$ & 0.3457469 & $\mathbf{0 , 7 0 2 6 8 7}$ \\
\hline 0.634787 & 0.3457469 & 1,0000 & \\
\hline
\end{tabular}

Таблица 4

Столбец №3 $Z^{(\ell, t)}{ }_{2}$ из МЛРА-выборки $Z_{m n}=\left[Z^{(t, \ell,)}{ }_{1} \mid Z^{(t, \ell)}{ }_{2}\right]$ при $\beta=(-0,347870,-0,225420)^{\mathrm{T}}$ (столбец №1) и при $\beta=(0.82920,0.702687)^{\mathrm{T}}$ (столбец №2), удовлетворяющие равенствам

$(1 \backslash \mathrm{m}) Z^{(t) T}{ }_{1} Z^{(t)}=R^{(\ell)}{ }_{11},(1 \backslash \mathrm{m}) Z^{(t) T}{ }_{1} Z^{(\ell, t)}{ }_{2}=R^{(\ell)}{ }_{12}, R^{(\ell)}{ }_{12}=R^{(\ell)}{ }_{11} \beta$,

\begin{tabular}{||r|r||}
\hline $\mathbf{j}=\mathbf{3}=>\left(\boldsymbol{\beta}_{\mathbf{1}}>\mathbf{0}, \boldsymbol{\beta}_{\mathbf{2}}<\mathbf{0}\right)$ & $\mathbf{j}=\mathbf{3}=>\left(\boldsymbol{\beta}_{\mathbf{1}}>\mathbf{0}, \boldsymbol{\beta}_{\mathbf{2}}>\mathbf{0}\right)$ \\
\hline 1 & 2 \\
\hline$-1,395987165$ & $-0,788634403$ \\
\hline 0,726717704 & 1,286619568 \\
\hline$-0,179229441$ & $-0,113674802$ \\
\hline
\end{tabular}




\begin{tabular}{|c|c|c|c|c|c|c|}
\hline Impact Factor: & $\begin{array}{l}\text { ISRA (India) } \\
\text { ISI (Dubai, UAE } \\
\text { GIF (Australia) } \\
\text { JIF }\end{array}$ & $\begin{array}{l}=1.344 \\
=0.829 \\
=0.564 \\
=1.500\end{array}$ & $\begin{array}{l}\text { SIS (USA) } \\
\text { PИНЦ (Russia) } \\
\text { ESJI (KZ) } \\
\text { SJIF (Morocco) }\end{array}$ & $\begin{array}{l}=0.912 \\
=0.156 \\
=4.102 \\
=5.667\end{array}$ & $\begin{array}{l}\text { ICV (Poland) } \\
\text { PIF (India) } \\
\text { IBI (India) }\end{array}$ & $\begin{array}{l}=6.630 \\
=1.940 \\
=4.260\end{array}$ \\
\hline
\end{tabular}

\begin{tabular}{||r|r||}
\hline$-1,050009996$ & $-0,367841477$ \\
\hline$-1,067333222$ & $-1,013006693$ \\
\hline$-0,521972316$ & $-0,727545649$ \\
\hline 0,645139770 & $-2,317663760$ \\
\hline 0,312486572 & $-0,145727144$ \\
\hline$-2,440672581$ & 0,285553966 \\
\hline 1,841886518 & 1,637375441 \\
\hline$-0,228296905$ & $-0,620842330$ \\
\hline$-0,337883618$ & $-0,894326873$ \\
\hline$-1,956154576$ & $-0,871726636$ \\
\hline 0,833235380 & 1,987463673 \\
\hline 0,117830072 & $-1,073202949$ \\
\hline 0,513217595 & 0,738557726 \\
\hline 0,230108734 & 0,418313570 \\
\hline$-0,245841581$ & $-0,245841581$ \\
\hline 0,123244747 & 0,123244747 \\
\hline$-0,143386205$ & $-0,143386205$ \\
\hline 1,000000000 & 1,000000000 \\
\hline
\end{tabular}

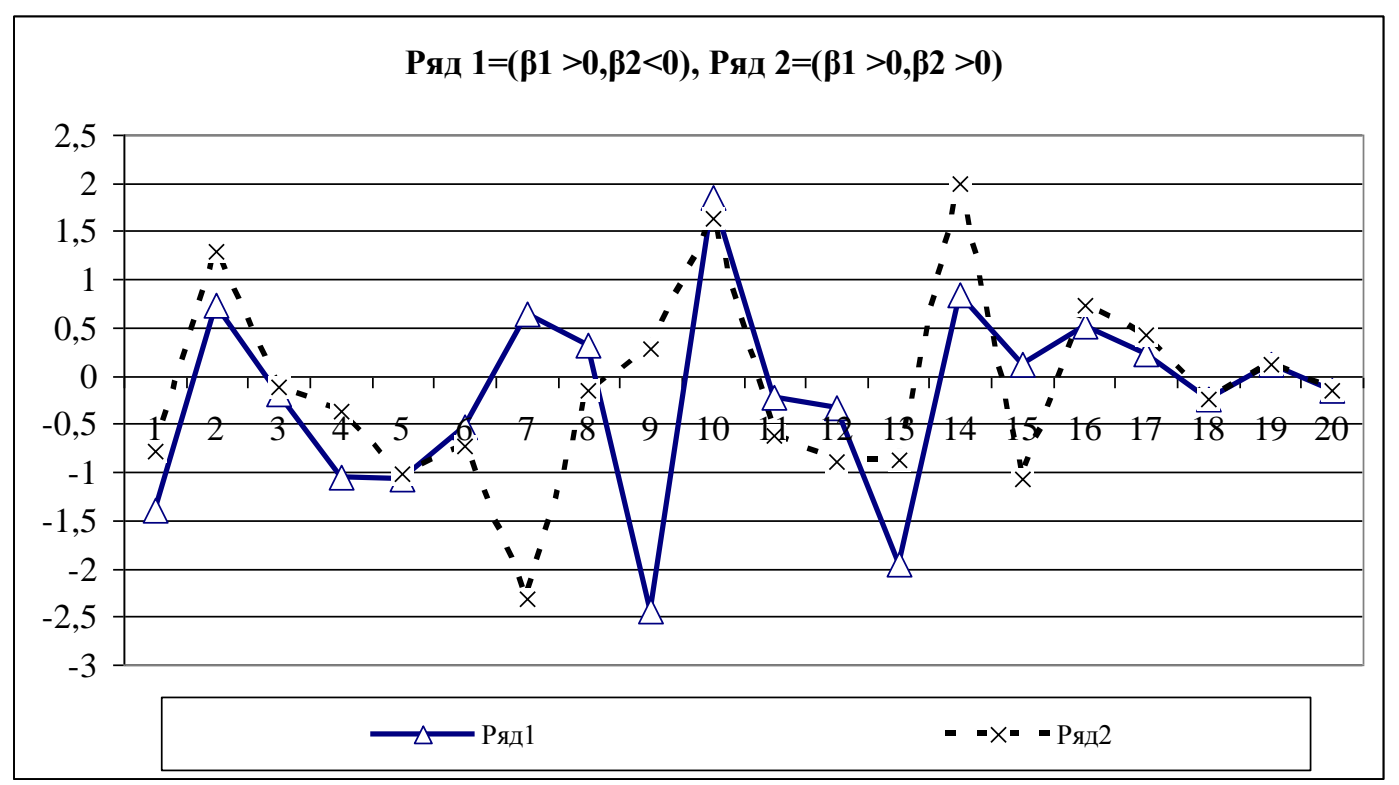

Рисунок 6 Динамики значений z-переменной №3, моделируемых в ОМ МЛРА а) при $\beta_{1}=0,634787, \beta_{2}=-0,42121$ (Ряд 1); б) при $\beta_{1}=0,82920, \beta_{2}=0,702687$ (Ряд 2) 


\begin{tabular}{l|lr|ll|ll} 
& ISRA (India) & $=\mathbf{1 . 3 4 4}$ & SIS (USA) & $=\mathbf{0 . 9 1 2}$ & ICV (Poland) & $=\mathbf{6 . 6 3 0}$ \\
Impact Factor: & ISI (Dubai, UAE) $=\mathbf{0 . 8 2 9}$ & PUHЦ (Russia) $=\mathbf{0 . 1 5 6}$ & PIF (India) & $=\mathbf{1 . 9 4 0}$ \\
& GIF (Australia) & $=\mathbf{0 . 5 6 4}$ & ESJI (KZ) & $=4.102$ & IBI (India) & $=\mathbf{4 . 2 6 0}$ \\
& JIF & $=\mathbf{1 . 5 0 0}$ & SJIF (Morocco) & $\mathbf{5 . 6 6 7}$ & & \\
\hline
\end{tabular}

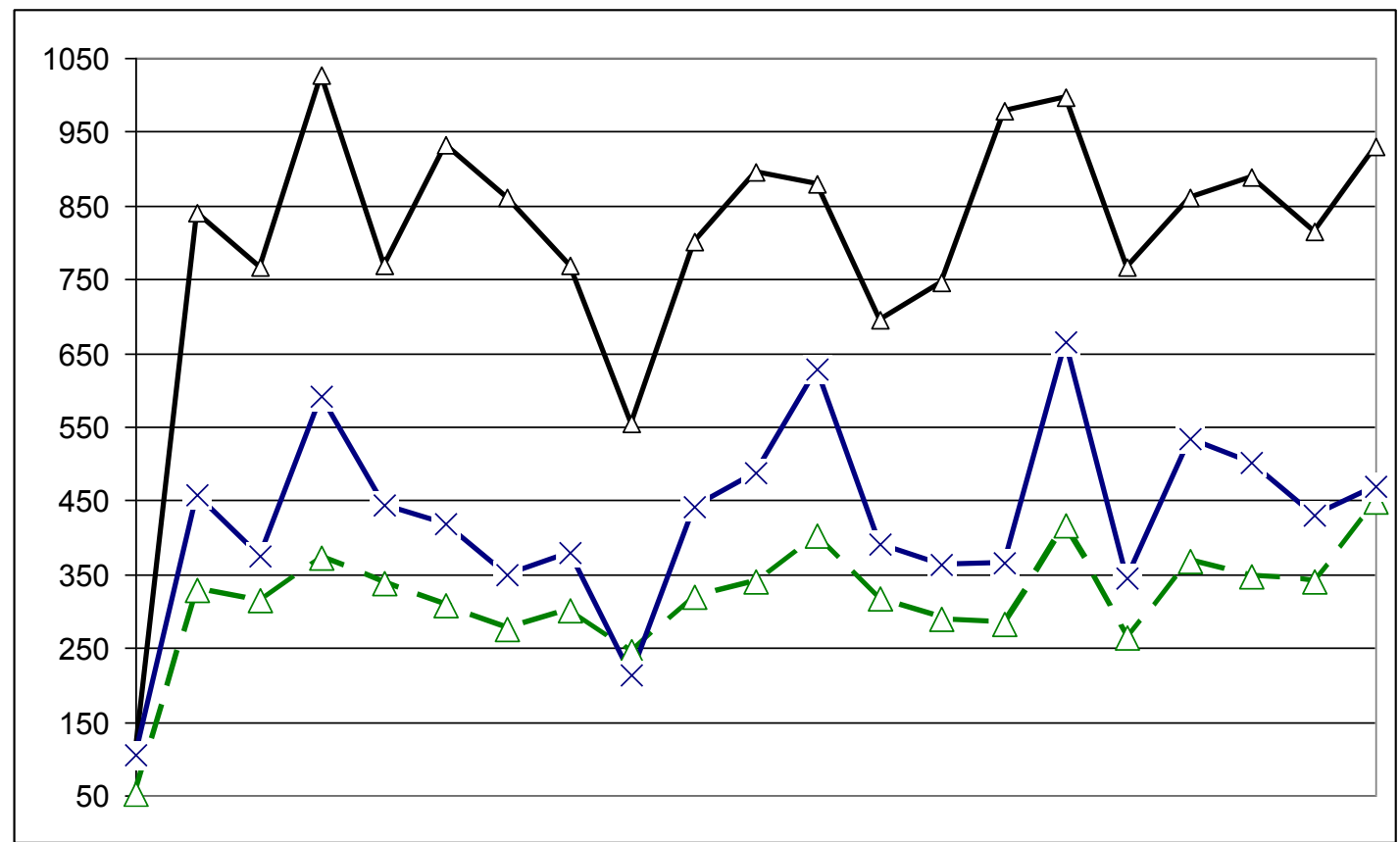

Рисунок 7 Динамики значений 3-x $\mathbf{x}^{0}$-переменных, преобразованных

из 3-х z-переменных, моделируемых в ОМ МЛРА при

$\beta_{1}=0.82920 ; \beta_{2}=0.702687, x_{1}{ }^{\mathrm{c}}=329.6, \mathrm{x}_{2}{ }^{\mathrm{cp}}=841.3, \mathrm{x}_{3}{ }^{\mathrm{c}}=456.9, \mathrm{~s}_{1}=51,1749, \mathrm{~s}_{2}=109,3621, \mathrm{~s}_{3}=105,1742$

\section{Заключение}

Мы конструировали ОМ МЛРА - обозначили постоянные величины (целые, вещественные) и переменные величины (детерминированные, случайные одномерные), воспользовались известными из ПМ МЛРА уравнениями, равенствами. Неизвестный вектор $\beta=\left(\beta_{1}, \ldots, \beta_{\mathrm{n}-1}\right)^{\mathrm{T}}$ коэффициентов регрессии из ПЗ МЛРА объявили в О3 МЛРА известным параметром. Постановки обратных задач (О3 АГК, О3 МЛРА) для известных популярных задач многомерной прикладной статистики - анализ главных компонент и множественный линейный регрессионный анализ, их задачи мы назвали прямыми задачами, являются новыми, а исследования с их применением: теоремы, задачи, приложения в других предметных областях, алгоритмы, компьютерные программы , результаты расчетов по реальным данным, выводы, цифровые знания, новые ключевые слова,- составляют новое научное направление в современной прикладной статистике. В этом направлении применена в данной статье сложная математическая модель ОМ МЛРА. Предстоит проводить глубокий анализ связанных с О3 МЛРА, с О3 АГК проблем. Исследовать обнаруженные новые явления, объекты, проявления их свойств и разработать алгоритмические методы работы с ними, выходить на новые рубежи знаний и технологийвиртуальные базы данных, виртуальные лаборатории. Чтобы успешно и всесторонне осмыслить существующие в этих моделях объекты, явления, процессы необходимо рассмотреть вопросы при переходе от безразмерных значений z-переменных к значениям $\mathrm{x}^{0}$-переменных (исходных переменных).

Псевдоадекватность имеет место при значениях средних арифметических независимых и одной n-1 зависимой переменной $\mathbf{x}_{\mathrm{cp}}=\left(\mathrm{x}_{1}{ }^{\mathrm{cp}}, \ldots, \mathrm{x}_{\mathrm{n}}\right.$ $\left.{ }_{1}{ }^{\mathrm{cp}}, \mathrm{x}_{\mathrm{n}}{ }^{\mathrm{cp}}\right)$, с их стандартными отклонениями $\mathrm{s}_{\mathrm{j}}=\left(\mathrm{x}^{2}{ }_{1 \mathrm{j}}+\ldots+\mathrm{x}^{2}{ }_{\mathrm{mj}}\right) / \mathrm{m}, \quad \mathrm{j}=1, \ldots, \mathrm{n}, \quad \mathrm{x}_{\mathrm{ij}}=\mathrm{x}_{\mathrm{ij}}{ }_{\mathrm{ij}}-\mathrm{x}_{\mathrm{j}}$ $\mathrm{i}=1, \ldots, \mathrm{m}, \mathrm{j}=1, \ldots, \mathrm{n}-1$. Это позволит оценивать приращения значения $\mathbf{x}_{\mathrm{n}}$ при заданном приращении одной из независимых $\mathrm{x}$ переменных. выходить на новые рубежи знаний и технологий.

Выше мы конструировали ОМ МЛРА обозначили постоянные величины (целые, вещественные) и переменные величины (детерминированные, случайные одномерные), воспользовались известными из ПМ МЛРА уравнениями, равенствами. Неизвестный вектор $\beta=\left(\beta_{1}, \ldots, \beta_{\mathrm{n}-1}\right)^{\mathrm{T}}$ коэффициентов регрессии из ПЗ МЛРА объявили в О3 МЛРА известным параметром. Постановки обратных задач (O3 АГК, ОЗ МЛРА) для известных популярных задач многомерной прикладной статистики - анализ главных компонент и множественный линейный регрессионный анализ, их задачи мы назвали прямыми задачами, являются новыми, а 
исследования с их применением: теоремы, задачи, приложения в других предметных областях, алгоритмы, компьютерные программы , результаты расчетов по реальным данным, выводы, цифровые знания,

Мы начали проводить глубокий анализ связанных с О3 МЛРА, с ОЗ АГК проблем. Исследо вать обнаруженные новые явления, объекты, проявления их свойств и разработать алгоритми ческие методы работы с ними, выходить на новые рубежи знаний и технологийвиртуальные базы данных, виртуальные лаборатории. Чтобы успешно и всесторонне осмыслить существующие в этих моделях объекты, явления, процессы необходимо рассмотреть вопросы при переходе от безразмерных значений z-переменных к значениям $\mathrm{x}^{0}$-переменных (исходных переменных). Здесь имеем дело с значениями средних арифметических независимых и одной n1 зависимой переменной $\mathbf{x}_{\mathrm{cp}}=\left(\mathrm{x}_{1}{ }^{\mathrm{cp}}, \ldots, \mathrm{x}_{\mathrm{n}-1}{ }^{\mathrm{cp}}, \mathrm{x}_{\mathrm{n}}{ }^{\mathrm{cp}}\right)_{9}, \mathrm{c}$ их стандартными отклонениями. Необходимо вычислять n-1 эластичностей переменной $\mathrm{x}_{\mathrm{n}}$ по хпеременным $\mathrm{x}_{1}, \ldots, \mathrm{x}_{\mathrm{n}}, \mathrm{x}_{\mathrm{ij}}=\mathrm{x}_{\mathrm{ij}}^{0}-\mathrm{x}_{\mathrm{j}}, \mathrm{i}=1, \ldots, \mathrm{m},=1, \ldots, \mathrm{n}$.

\section{References:}

1. Zhanatauov SU. (2018) Inverse model of multiple linear regression analysis. International scientific journal Theoretical \&Applied Science.2018, №4(60):201-212. www.T-Science.org.

2. Zhanatauov SU. (2018) Model of digitalization of the validity indicators and of the measurable indicatojrs of the enterprise. International scientific journal Theoretical \&Applied Science.2018, №9(65): www.T-Science.org.

3. Kas"yanenko T.E. (2011) Preobrazovanie finansovoy otchet nosti predpriyatya dlya tseley otsenki biznesa. Izd-vo Sank-Peterburgskogo gosudarstvennogo Uni versiteta ekonomiki i finansov.2011, $169 \mathrm{p}$

4. Hotelling H. (1933) Analysis of a complex of statistical variables into principal compo nents.J.Educ. Psychol. ,1933, vol.24, p. 417-441, p. 498-520.

5. Zhanatauov S.U. (1987) Obratnaya model' glavnykh kompo nent i ee primenenie. Diss. na soiskanie uch. step.. kand. fiz.-mat. nauk:05.13.11:zashchishchena 8.12.1987: utv.1.06.1988/ZhanatauovSapargaliUtepovichVychis litel'nyy tsentr Sibirskogo otdeleniya AN SSSR, Novosibirsk, 1987g., 302 p.

6. Zhanatauov S.U. (1989) Modelirovanie odnoy zamechatel' noy ekstremal'noy sovokupnosti// Sistemnoe modeli rovanie-14, Novosibirsk.1989. p.27- 33.

7. ZhanatauovS.U. (2013) Obratnaya model' glavnykh kompo nent:-monografiya.-Almaty: Kazstatinform, 2013.-201 p.

8. Zhanatauov S.U. (2017) A model of calculation risk changing of the interest rate "yield to maturity date" for foreign currency bonds of the republic of Kazakhstan. International scientific journal Theoretical\& Applied
Science.2017,№8,vol.52,rr.19-36.www.T-

Science.org.

9. Zhanatauov SU. (2017) Theorem on the $\Lambda$ samples. Inter national scientific journal Theoretical\&Applied Science. 2017,№ 9,vol.53, rr.177-192. www.T-Science.org.

10. Zhanatauov SU (2017) Optimization problem of modeling missing elements of the spectrum of the correlation matrix. International scientific journal Theoretical \&Applied Science. 2017,№10, vol.54,rr.189-198. w.TScience.org.

11. Zhanatauov SU (2017) .The optimization problem with linearized equations f-parameters (f1,f2,f3,f4,f5,f6)-spectrum. International scientific journal Theoretical \&Applied Science. 2017,№11,vol.55,p.251-267. www.TScience.org.

12. Zhanatauov S.U. (2016) Model and histogram to adequacy of variables $(\mathrm{C}, \Lambda)$-samples and real multidimensional sample. International Scientific Journal Theoretical \&Applied Science.2016, № 11, vol. 43, p. 53-61. www.TScience.org.

13. Zhanatauov S.U. (2014) The $(\mathrm{C}, \Lambda, Y)$-sample is adequate to real multidimensional sample. Proced. Int. conf.. "Leadership in Education, Business and Culture". 25 apriel 2014, AlmatySeatle, ICET USA. Leadership Iternational Conference "Leadership on Education, Business and Culture». p.151-155.

14. Zhanatauov S.U. (2017) Modelirovanie mnogomernykh vyborok znacheniy priznakov zernovoy kul'tury. "II mezhdun. nauchnoprakt.konf. «Evropa i tyurkskiy mir: nauka, tekhnika i tekhnologii".Izmir (Turtsiya), 29-31 maya 2017. www.regionacadem.org. 


\begin{tabular}{l|lr|ll|ll} 
& ISRA (India) & $=\mathbf{1 . 3 4 4}$ & SIS (USA) & $=\mathbf{0 . 9 1 2}$ & ICV (Poland) & $=\mathbf{6 . 6 3 0}$ \\
Impact Factor: & ISI (Dubai, UAE) $=\mathbf{0 . 8 2 9}$ & PUHЦ (Russia) $=\mathbf{0 . 1 5 6}$ & PIF (India) & $=\mathbf{1 . 9 4 0}$ \\
& GIF (Australia) & $=\mathbf{0 . 5 6 4}$ & ESJI (KZ) & $=4.102$ & IBI (India) & $=\mathbf{4 . 2 6 0}$ \\
& JIF & $=\mathbf{1 . 5 0 0}$ & SJIF (Morocco) & $=2.031$ & & \\
\hline
\end{tabular}

15. ZhanatauovS,U.O (1988) funktsional'nom napolnenii PPP "Spektr".Sistemnoe modelirovanie-13.-Novosibirsk, 1988, p.3-11.

16. Zhanatauov S.U. (1987) The inverse problem of the principal component analysis// Proc.of the 1-st World Congress of Soc. Math. Statist. and
Probabillity Theory of Bernoulli. - Utrecht, 1987. - p. 116-119.

17. Zhanatauov S.U. (1980) Metod polucheniya vyborki s zadannymi sobstvennymi chislami ee korrelyatsion noy matritsy. - V kn. Matematicheskie voprosy analiza dannykh. Novosibirsk, 1980, p.62-76. 